TRANSACTIONS OF THE

AMERICAN MATHEMATICAL SOCIETY

Volume 354, Number 6, Pages 2369-2397

S 0002-9947(02)02955-0

Article electronically published on February 4, 2002

\title{
TWISTED FACE-PAIRING 3-MANIFOLDS
}

\author{
J. W. CANNON, W. J. FLOYD, AND W. R. PARRY
}

\begin{abstract}
This paper is an enriched version of our introductory paper on twisted face-pairing 3-manifolds. Just as every edge-pairing of a 2-dimensional disk yields a closed 2-manifold, so also every face-pairing $\epsilon$ of a faceted 3ball $P$ yields a closed 3 -dimensional pseudomanifold. In dimension 3 , the pseudomanifold may suffer from the defect that it fails to be a true 3-manifold at some of its vertices. The method of twisted face-pairing shows how to correct this defect of the quotient pseudomanifold $P / \epsilon$ systematically. The method describes how to modify $P$ by edge subdivision and how to modify any orientation-reversing face-pairing $\epsilon$ of $P$ by twisting, so as to yield an infinite parametrized family of face-pairings $(Q, \delta)$ whose quotient complexes $Q / \delta$ are all closed orientable 3-manifolds. The method is so efficient that, starting even with almost trivial face-pairings $\epsilon$, it yields a rich family of highly nontrivial, yet relatively simple, 3-manifolds.
\end{abstract}

This paper solves two problems raised by the introductory paper:

(1) Replace the computational proof of the introductory paper by a conceptual geometric proof of the fact that the quotient complex $Q / \delta$ of a twisted face-pairing is a closed 3-manifold. We do so by showing that the quotient complex has just one vertex and that its link is the faceted sphere dual to $Q$.

(2) The twist construction has an ambiguity which allows one to twist all faces clockwise or to twist all faces counterclockwise. The fundamental groups of the two resulting quotient complexes are not at all obviously isomorphic. Are the two manifolds the same, or are they distinct?

We prove the highly nonobvious fact that clockwise twists and counterclockwise twists yield the same manifold. The homeomorphism between them is a duality homeomorphism which reverses orientation and interchanges natural 0-handles with 3-handles, natural 1-handles with 2-handles. This duality result of (2) is central to our further studies of twisted face-pairings.

We also relate the fundamental groups and homology groups of the twisted face-pairing 3-manifolds $Q / \delta$ and of the original pseudomanifold $P / \epsilon$ (with vertices removed).

We conclude the paper by giving examples of twisted face-pairing 3-manifolds. These examples include manifolds from five of Thurston's eight 3dimensional geometries.

Received by the editors December 8, 2000 and, in revised form, October 5, 2001.

2000 Mathematics Subject Classification. Primary 57Mxx.

Key words and phrases. 3-manifold constructions, surgeries on 3-manifolds, Thurston's geometries.

This research was supported in part by NSF grants DMS-9803868, DMS-9971783, and DMS10104030.

(C)2002 American Mathematical Society 


\section{INTRODUCTION}

The twisted face-pairing construction, introduced in [4, takes as input an arbitrary orientation-reversing face-pairing $\epsilon$ of a faceted 3-ball $P$ (see definitions in Section 2), and returns an infinite, parametrized family of orientation-reversing facepairings $(Q, \delta)$ for which every quotient $Q / \delta$ is a closed, orientable 3-manifold. Even when the original face-pairing $(P, \epsilon)$ is very simple, or even trivial, the associated face-pairings $(Q, \delta)$ are almost always interesting and nontrivial, yet have relatively simple face-pairing descriptions, and have fundamental groups whose natural presentations are balanced with long relators. Thus twisted face-pairing 3-manifolds supply a wonderful and rich field of exploration for classroom use, and supply an incomparable source for those of us who study 3-manifolds in terms of the asymptotic combinatorial properties of their universal covers. Anyone who has sought relatively simple face-pairing descriptions of interesting 3-manifolds will recognize the difficulties resolved by this construction.

We review the construction here. Given an orientation-reversing face-pairing $\epsilon$ on a faceted 3-ball $P$ and a face $f$ of $P$, let $\epsilon_{f}$ denote the cellular homeomorphism by which $\epsilon$ identifies $f$ with its paired face. Let $\sim$ be the equivalence relation on the set of edges of $P$ generated by $e \sim \epsilon_{f}(e)$ when $e$ is an edge of $f$. We call the resulting equivalence classes edge cycles. For each edge cycle $[e]$, let $\ell_{[e]}$ be the number of edges in $[e]$ and let $m_{[e]}$ be a positive integer. We call $m_{[e]}$ the multiplier of $[e]$ and denote the function $[e] \mapsto m_{[e]}$ by mul. We obtain a cell complex $Q=Q(\epsilon$, mul $)$ from $P$ by subdividing every edge $e$ into $\ell[e] m[e]$ subedges. Given a face $f$ of $Q$, we construct an orientation-preserving cellular homeomorphism $\tau_{f}: f \rightarrow f$ that takes each vertex $v$ of $f$ to the vertex of $f$ that follows $v$ in the orientation on $\partial f$ induced from the orientation on $Q$. We then obtain a face-pairing $\delta$ on $Q$ by letting $\delta_{f}=\epsilon_{f} \circ \tau_{f}$, and we denote by $M=M(\epsilon$, mul $)$ the quotient complex $Q / \delta$. The fundamental theorem of twisted face-pairings is that $M$ is always a 3 -manifold. This was proved in [4 by an Euler characteristic argument. Our first goal in this paper will be to give a conceptual geometric proof of the same result. We do so by showing that the quotient complex has just one vertex and that its link is isomorphic with the faceted sphere dual to $\partial(Q)$ (Theorem 3.1). (Actually, when we are careful about orientations, we shall see that this isomorphism reverses orientations. We shall discuss orientations a bit more in the next paragraph.) We call $M$ a twisted face-pairing 3-manifold.

Note that the direction in which $\epsilon$ is twisted in forming the new face-pairing $\delta$ is determined by the orientation of $Q$, which is determined by the original given orientation on $P$. But $P$ (and $Q$ ) have two orientations, so that $\delta$ could have been defined so as to twist in the opposite direction. For our purposes, the natural way to encode this opposite twist is to denote by $P^{*}$ and by $Q^{*}$ the faceted 3-balls $P$ and $Q$ with the opposite orientation. Then $\left(Q^{*}, \delta^{*}\right)$ denotes the twisted face-pairing resulting from twists in the opposite direction. Our main result in Section 4 is the proof of the highly nonobvious fact that $Q / \delta$ and $Q^{*} / \delta^{*}$ give dual cell structures of the same manifold. This material is crucial for the further development of the theory that will appear in papers [5] and [6].

In Section 5 , we show, among other things, that there is an epimorphism $\pi_{1}(M)$ $\rightarrow \pi_{1}(P / \epsilon)$, where $\pi_{1}(P / \epsilon)$ is taken to mean the fundamental group of $P / \epsilon$ with vertices removed. The homology groups of $M$ and $P / \epsilon$ are compared in Section 6 . We conclude the paper in Section 7 with examples. The examples include manifolds 
from five of the eight 3-dimensional geometries. In 6 we use a more general cellulation of $P$ to give a twisted face-pairing 3-manifold with geometry $S^{2} \times \mathbf{R}$. We know of no twisted face-pairing 3-manifold which has one of the product geometries $\mathbf{E}^{3}$ or $\mathbf{H}^{2} \times \mathbf{R}$.

This paper lays the groundwork for further papers, including [5], 6], and [7]. We say that a faceted 3 -ball $P$ is ample if it satisfies the following conditions:

i) if two distinct faces of $P$ intersect, then they intersect in a vertex or an edge;

ii) if three distinct faces of $P$ intersect pairwise, then their common intersection is a vertex;

iii) no face of $P$ is a triangle.

In [5] we show that if $P$ is an ample faceted 3-ball, $\epsilon$ is an orientation-reversing facepairing on $P$, and mul is a multiplier function for $\epsilon$, then $\pi_{1}(M(\epsilon, \mathrm{mul}))$ is Gromov hyperbolic with space at infinity a 2-sphere. Hence one can use this procedure to easily construct 3-manifolds with Gromov hyperbolic fundamental groups. It is hoped that these will be useful test examples for our approach (see, for example, [1], [2], 3] and [8]) to Thurston's Hyperbolization Conjecture. Indeed, the search for such examples was the primary motivation for the development of this twisted face-pairing construction.

In [6] we describe twisted face-pairing 3-manifolds in terms of Heegaard diagrams and Dehn surgeries on framed links. Suppose we are given a faceted 3-ball $P$ with $2 n$ faces and an orientation-reversing face-pairing $\epsilon$ on $P$. Let $E_{1}, \ldots, E_{m}$ be the edge cycles. Let $S$ be a closed orientable surface of genus $n$, and let $\left\{\alpha_{1}, \ldots, \alpha_{n}\right\}$ be a family of nontrivial, pairwise disjoint, simple closed curves on $S$ whose union does not disconnect $S$. Then there is a family $\left\{\beta_{1}, \ldots, \beta_{m}\right\}$ of pairwise disjoint simple closed curves on $S$ with the following property. For each $i \in\{1, \ldots, m\}$, let $\tau_{i}$ be one of the two Dehn twists on $S$ about $\beta_{i}$, chosen so that the directions in which we twist are consistent. Given a multiplier function mul for $\epsilon$, let $\tau^{\text {mul }}=$ $\tau_{1}^{\operatorname{mul}\left(E_{1}\right)} \circ \cdots \circ \tau_{m}^{\operatorname{mul}\left(E_{m}\right)}$. We prove that $S$ and the two families of curves $\left\{\alpha_{1}, \ldots, \alpha_{n}\right\}$ and $\left\{\tau^{\mathrm{mul}}\left(\alpha_{1}\right), \ldots, \tau^{\mathrm{mul}}\left(\alpha_{n}\right)\right\}$ form a Heegaard diagram for $M(\epsilon, \mathrm{mul})$. The surgery description comes naturally from this. From $P$ and $\epsilon$ we get a diagram of a link in $S^{3}$ with components $\gamma_{1}, \ldots, \gamma_{n}, \delta_{1}, \ldots, \delta_{m}$. Then $M(\epsilon$, mul $)$ is obtained by Dehn surgery from this link with framing 0 for each $\gamma_{i}$ and framing $1 / \operatorname{mul}\left(E_{j}\right)$ plus the blackboard framing for each $\delta_{j}$.

From the point of view of producing a single manifold $M$, there is no need to have a multiplier function. For suppose we are given a faceted 3-ball $P$ with orientationreversing face-pairing $\epsilon$ and multiplier function mul. Let $P^{\prime}$ be the faceted 3-ball obtained from $P$ by subdividing each edge $e$ of $P$ into $m_{[e]}$ subedges. Then $\epsilon$ induces a face-pairing $\epsilon^{\prime}$ on $P^{\prime}$, and $M(\epsilon, \mathrm{mul}) \cong M\left(\epsilon^{\prime}, \mathrm{mul}^{\prime}\right)$, where mul' is the constant multiplier function which assigns 1 to each edge cycle of $P^{\prime}$. So by replacing $P$ by $P^{\prime}$ one could assume that each multiplier is 1 . However, there are advantages to allowing and using multiplier functions. If $P$ is an ample faceted 3-ball with orientation-reversing face-pairing $\epsilon$ and multiplier function mul, then the associated faceted 3-ball $P^{\prime}$ will not be ample if any multiplier is greater than one. So by switching to $P^{\prime}$ one would not be able to conclude from [5] that $\pi_{1}(M)$ is Gromov hyperbolic. Furthermore, as discussed in the previous paragraph, multipliers are inverses of framings of link components and by using multipliers one can often give unified treatments of infinite families of examples. 


\section{Primary Definitions}

We begin with a cellulation of a 3 -ball. We have in mind a polyhedron in that word's original meaning. Since the word polyhedron has come to mean something much more general, we instead use the expression faceted 3-ball. So we take a faceted 3-ball to be a cellulation of a 3-ball with exactly one 3-cell. We next must make the term cellulation precise. There is not an obvious class of cell complexes on the 2-sphere for us to use. We might work with more general cell complexes, but we find the class of cell complexes which we choose here to be interesting and convenient, so we proceed as follows.

A faceted 3-ball $P$ is an oriented regular CW complex such that $|P|$ is a closed 3 -ball and $P$ has a single 3 -cell. The regularity condition means that for each open cell the prescribed homeomorphism of an open Euclidean ball to that cell extends to a homeomorphism of the closed Euclidean ball to the closed cell. If $P$ is a faceted 3-ball, a face-pairing $\epsilon$ on $P$ consists of the following. First, the faces of $P$ are paired: for every face $f$ of $P$ there exists a face $f^{-1} \neq f$ of $P$ such that $\left(f^{-1}\right)^{-1}=f$. Second, for every face $f$ of $P$ there exists a cellular homeomorphism $\epsilon_{f}: f \rightarrow f^{-1}$ called a face-pairing map such that $\epsilon_{f^{-1}}=\epsilon_{f}^{-1}$. We furthermore impose a compatibility condition on the face-pairing maps two paragraphs below. Set $\epsilon=\left\{\epsilon_{f}: f\right.$ is a face of $\left.P\right\}$. We say that $\epsilon$ is orientation-reversing if and only if every face-pairing map reverses orientation.

Suppose $P$ is a faceted 3 -ball and $\epsilon$ is an orientation-reversing face-pairing on $P$. Let $\sim$ be the least equivalence relation on the set of edges of $P$ such that, if $f$ is a face of $P$ and $e$ is an edge of $f$, then $e \sim \epsilon_{f}(e)$. The equivalence classes of $\sim$ are called edge cycles. We draw diagrams of edge cycles as follows. Let $e_{1}, \ldots, e_{j}$ be the distinct edges of an edge cycle $E$ so that for every $i \in\{1, \ldots, j\}$ there exists a face $f_{i}$ of $P$ with $e_{i} \subseteq f_{i}$ and $e_{i+1}=\epsilon_{f_{i}}\left(e_{i}\right)$, where the indices are taken modulo $j$. We draw the following diagram:

$$
e_{1} \stackrel{\epsilon_{f_{1}}}{\rightarrow} e_{2} \stackrel{\epsilon_{f_{2}}}{\rightarrow} \cdots \stackrel{\epsilon_{f_{j-1}}}{\rightarrow} e_{j} \stackrel{\epsilon_{f_{j}}}{\rightarrow} e_{1} .
$$

For each edge cycle $E$, let $\ell_{E}$ be the number of edges in $E$ and let $m_{E}$ be a positive integer. We call $\ell_{E}$ the length of the edge cycle $E$. The function mul : \{edge cycles\} $\rightarrow \mathbf{N}$ defined by $E \mapsto m_{E}$ is called the multiplier function, and $m_{E}$ is called the multiplier of $E$.

As on page 123 of [11], to ensure that the quotient space $P / \epsilon$ is a cell complex, we require that our face-pairing maps satisfy the following face-pairing compatibility condition. Every edge cycle diagram as in line 2.1 in effect represents a composition of functions, these functions being face-pairing maps restricted to edges. We require that the composition of functions arising from every edge cycle diagram be the identity map. Hence for the edge cycle diagram in (2.1) we require that

$$
\left.\left.\left.\epsilon_{f_{j}}\right|_{e_{j}} \circ \cdots \circ \epsilon_{f_{2}}\right|_{e_{2}} \circ \epsilon_{f_{1}}\right|_{e_{1}}
$$

be the identity map on $e_{1}$. As in [11, Problem 3.2.10], it follows that the action of the face-pairing maps on vertices determines $P / \epsilon$ up to homeomorphism.

Now construct a faceted 3-ball $Q=Q(\epsilon$, mul $)$ from $P$ by just subdividing the edges of $P$ as follows. Let $e$ be an edge of $P$, and let $E$ be the edge cycle of $e$. We subdivide $e$ into $\ell_{E} m_{E}$ subedges. The face-pairing compatibility condition implies that we may choose this subdivision in an $\epsilon$-invariant way: if $v$ is a vertex of $Q$ 


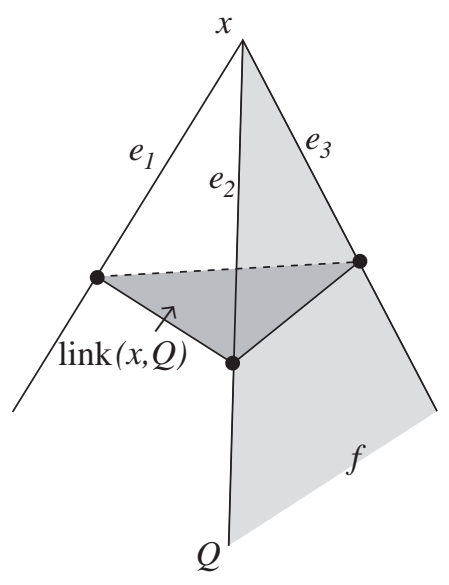

Figure 1. The link of $x$ in $Q$.

contained in a face $f$ of $P$, then $\epsilon_{f}(v)$ is also a vertex of $Q$. Every vertex of $P$ determines a vertex of $Q$, called an original vertex of $Q$. Similarly, every edge of $P$ determines a subcomplex of $Q$, called an original edge of $Q$. The vertices of $Q$ which are not original vertices are called new vertices.

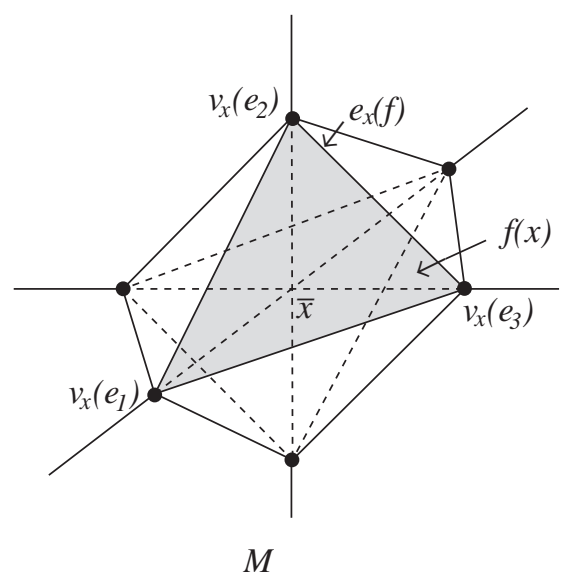

Figure 2. The link of $\bar{x}$ in $M$.

We orient $Q$ so that the identity map $|P| \rightarrow|Q|$ is orientation preserving. The orientations on $P$ and $Q$ induce orientations on each face $f$ of $P$ or $Q$, and these in turn determine orientations on the boundary $\partial f$ of each face $f$. The definition of faceted 3-ball assures that given a face $f$ of $P$ or $Q$ and a vertex $x$ of $f$, the notion of following vertex relative to $f$ is meaningful: it is the vertex $y$ of $f$ adjacent to $x$ in the direction of the orientation of $\partial f$. We say that $x$ is the vertex preceding $y$ relative to $f$. Similarly, we have preceding and following edges and original edges relative to $f$. The edges of $f$ in a given original edge of $f$ are ordered relative to $f$.

Because we obtain $Q$ by subdividing $P$ in an $\epsilon$-invariant way, the face-pairing $\epsilon$ on $P$ naturally determines a face-pairing, which we continue to call $\epsilon$, on $Q$. For 


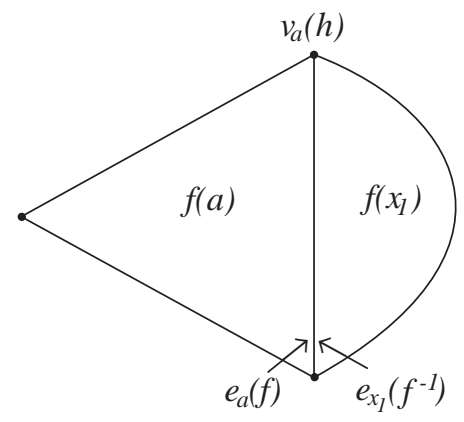

Figure 3. Two faces of $S$.

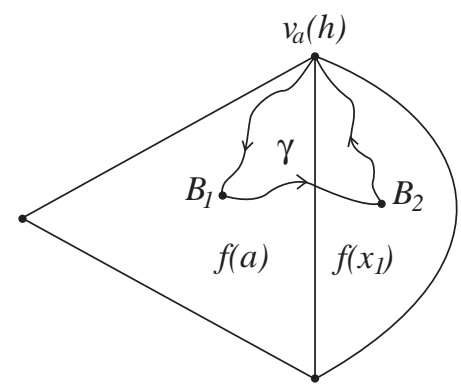

Figure 4 . The simple closed curve $\gamma$.

each face $f$ of $Q$, let $\tau_{f}$ be an orientation-preserving cellular homeomorphism of $f$ which takes each vertex of $f$ to its following vertex. We assume that these maps are defined $\epsilon$-invariantly; that is, for each face $f$ of $Q$ we have $\tau_{f^{-1}}=\epsilon_{f} \circ \tau_{f}^{-1} \circ \epsilon_{f^{-1}}$. Let $\delta$ be the face-pairing on $Q$ with the same pairing of faces as for the face-pairing $\epsilon$, and with $\delta_{f}=\epsilon_{f} \circ \tau_{f}$ for each face $f$ of $Q$. We assume that the maps $\tau_{f}$ are defined so that $\delta$ satisfies the face-pairing compatibility condition. Then $\delta$ is a face-pairing on $Q$ called the twisted face-pairing. We refer to $P$ as the model faceted 3-ball and we refer to $\epsilon$ as the model face-pairing from which $Q$ and $\delta$ are constructed.

Define $M=M(\epsilon$, mul $)$ to be the cell complex $Q / \delta$ consisting of orbits of points of $Q$ under $\delta$. We show in Theorem 3.1 that $M$ is always a manifold.

By a dual of a faceted 3-ball $P$ we mean a faceted 3-ball for which there exists an orientation-reversing cellular homeomorphism to $P$. Given a faceted 3-ball $P$, we let $P^{*}$ denote a faceted 3-ball dual to $P$. Given $P, \epsilon$, mul, and $Q$ as above, we fix an orientation-reversing cellular homeomorphism $\omega: Q \rightarrow Q^{*}$. Notions such as original vertex and edge extend to $Q^{*}$.

Let $x$ be a vertex of $Q$, and denote by $\bar{x}$ the image of $x$ in $M$. Then $\operatorname{link}(x, Q)$ is canonically isomorphic to a face $f(x)$ of $\operatorname{link}(\bar{x}, M)$. If $f$ is a face of $Q$ containing $x$, then $f$ determines an edge $e_{x}(f)$ of $\operatorname{link}(\bar{x}, M)$. Similarly, if $e$ is an edge of $Q$ containing $x$, then $e$ determines a vertex $v_{x}(e)$ of $\operatorname{link}(\bar{x}, M)$. See Figures 1 and 2 .

We use the clockwise orientation of faces in all figures. 


\section{Fundamental THEOREM}

The following is the fundamental theorem of twisted face-pairing. We use the notation and terminology of the previous section.

Theorem 3.1. Let $P$ be a faceted 3-ball, let $\epsilon$ be an orientation-reversing facepairing on $P$, and let mul be a multiplier function for $\epsilon$. Then the cell complex $M=M(\epsilon, m u l)$ is an orientable closed 3-dimensional manifold. Furthermore, $M$ has just one vertex, $u$, and the dual of $\operatorname{link}(u, M)$ is isomorphic to $\partial Q^{*}$ as oriented 2-complexes. This isomorphism is determined by the map $\omega: Q \rightarrow Q^{*}$; every face of link $(u, M)$ is canonically isomorphic to link $(x, Q)$ for some unique vertex $x$ of $Q$ and the barycenter of this face corresponds to the vertex $\omega(x)$ of $Q^{*}$.

Proof. Our argument proceeds by first proving the last two sentences of the theorem. Once this is done, it follows that the link of every vertex of $M$ is a 2-sphere, and so $M$ is a manifold by a well-known theorem. For example, see [9, §59, Theorem 1] or [11, Prop. 3.2.7].

Let $f$ be a face of $Q$, let $a$ be an original vertex of $f$, and let $h$ be the edge of $f$ preceding $a$. We investigate the star $S$ of $v_{a}(h)$ in $\operatorname{link}(\bar{a}, M)$.

For this, let $x_{1}=\delta_{f}(a)$ and let $h_{1}=\delta_{f}(h)$. It follows that 1) $\left.\overline{x_{1}}=\bar{a}, 2\right) v_{x_{1}}\left(h_{1}\right)=$ $v_{a}(h)$ and 3) $e_{x_{1}}\left(f^{-1}\right)=e_{a}(f)$. See Figure 3. Thus $f(a)$ and $f\left(x_{1}\right)$ are faces of $S$ which share the edge $e_{x_{1}}\left(f^{-1}\right)=e_{a}(f)$ which contains $v_{a}(h)$. Furthermore, because $h$ is the edge of $f$ preceding $a$, if $B_{1}$ is a barycenter of $f(a)$ and $B_{2}$ is a barycenter of $f\left(x_{1}\right)$ and $\gamma$ is a simple closed curve from $v_{a}(h)$ to $B_{1}$ to $B_{2}$ to $v_{a}(h)$ as shown in Figure 4, then $\gamma$ is oriented in the direction opposite to the orientation of the faces of $Q$. (Recall that all figures are drawn using the clockwise orientation for the faces of $Q$.)

We use Figure 5 to continue the investigation of $S$. Let $a_{1}=a$, let $b_{1}$ be the original vertex of $f$ following $a$ and let $e_{1}$ be the original edge of $f$ following $a$, so that $a_{1}$ and $b_{1}$ are the endpoints of $e_{1}$. Let $f_{1}=f$. We maintain the meaning of $x_{1}$ and $h_{1}$ in the previous paragraph. The edge cycle $E$ of the edge of $P$ corresponding to $e_{1}$ is depicted in Figure [5. Fix $k \in\left\{1, \ldots, \ell_{E} m_{E}-1\right\}$ for the rest of this paragraph. Suppose that $a_{k}, b_{k}, e_{k}, f_{k}, x_{k}$, and $h_{k}$ are defined. Define $a_{k+1}, b_{k+1}$, $e_{k+1}$, and $f_{k+1}$ so that $a_{k+1}=\epsilon_{f_{k}}\left(a_{k}\right), b_{k+1}=\epsilon_{f_{k}}\left(b_{k}\right), e_{k+1}=\epsilon_{f_{k}}\left(e_{k}\right)$ and $f_{k+1}$ is the unique face of $Q$ other than $f_{k}^{-1}$ which contains $e_{k+1}$. Note that $a_{k}, b_{k}, e_{k}$ and $f_{k}$ are periodic with period $\ell_{E}$. Define $x_{k+1}$ and $h_{k+1}$ so that $x_{k+1}=\delta_{f_{k+1}}\left(x_{k}\right)$ and $h_{k+1}=\delta_{f_{k+1}}\left(h_{k}\right)$. Then $h_{k}$ is the $k$ th edge of $e_{k+1}$ relative to $f_{k+1}$ and $h_{\ell_{E} m_{E}}$ is the last edge of $e_{1}$ relative to $f_{1}$. In particular, $x_{\ell_{E} m_{E}}=b_{1}$. Just as in the previous paragraph, we see that $f\left(x_{k}\right)$ and $f\left(x_{k+1}\right)$ are faces of $S$ which share the edge $e_{x_{k}}\left(f_{k+1}\right)$ which contains $v_{a}(h)$. See Figure [6. Since the vertices $x_{1}, \ldots, x_{\ell_{E} m_{E}-1}$ have valence 2 , the faces $f\left(x_{1}\right), \ldots, f\left(x_{\ell_{E} m_{E}-1}\right)$ are digons.

We now repeat the discussion of the previous three paragraphs, replacing $h$ by $h_{\ell_{E} m_{E}}$ and replacing $a_{1}$ by $b_{1}$. Continuing, we see that the dual of the link of $v_{a}(h)$ in $S$ is canonically isomorphic to $\partial f$. Furthermore, the discussion of the simple closed curve $\gamma$ in Figure 4 shows that as we traverse $\partial f$ in the positive direction, we traverse the dual of the link of $v_{a}(h)$ in $S$ in the opposite direction.

Since the conclusions of the previous paragraph hold for every face of $Q$, it easily follows that $M$ has just one vertex and all of the assertions of Theorem 3.1 are true.

This proves Theorem 3.1.

We call $M$ a twisted face-pairing 3-manifold. 


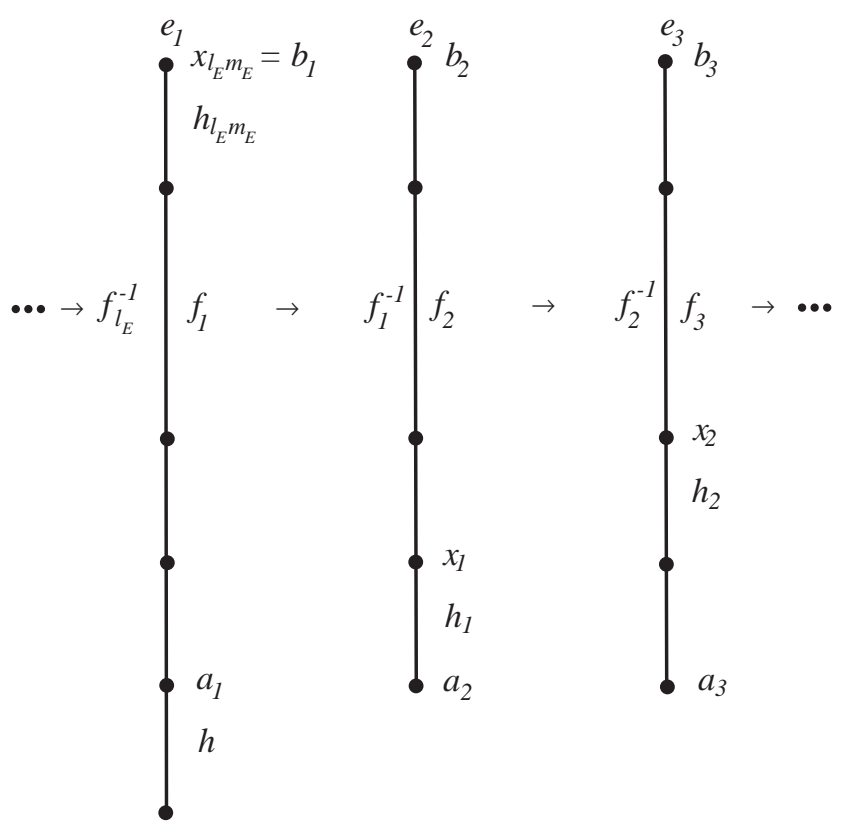

Figure 5. Defining $a_{k}, b_{k}, e_{k}, f_{k}, x_{k}$, and $h_{k}$.

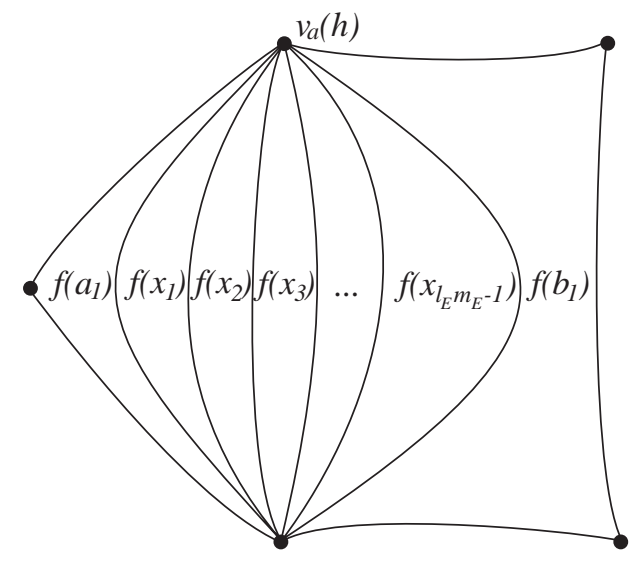

Figure 6 . The part of the link of the vertex of $M$ corresponding to the original edge of $f$ from $a_{1}$ to $b_{1}$.

We wish to label and direct every face of $P$ and $Q$. This direction of faces is not to be confused with the orientation of faces induced by the orientation of $P$ and $Q$. For this, suppose that $Q$ has $n$ pairs of faces, and let $F_{1}, \ldots, F_{n}$ be representatives of these pairs, so that the faces of $Q$ are $F_{1}^{ \pm 1}, \ldots, F_{n}^{ \pm 1}$. Let $f$ be a face of $Q$. Then there exists a unique $i \in\{1, \ldots, n\}$ such that $f \in\left\{F_{i}, F_{i}^{-1}\right\}$. We label $f$ with $i$. Suppose $f=F_{i}$. In this case we say that $f$ is directed outward. By this we have in mind a vector normal to $f$ pointing out of $Q$. Similarly, if $f=F_{i}^{-1}$, then we say that $f$ is directed inward, and we have in mind a vector normal to $f$ pointing 
into $Q$. We label and direct the faces of $P$ so that the canonical correspondence between the faces of $P$ and $Q$ respects labels and directions. If we label a face of $P$ or $Q$ in a figure with $i^{\prime}$, then that face has label $i$ and is directed inward; a face of $P$ or $Q$ in a figure with label simply $i$ is understood to be directed outward. If $f$ is a face of $P$ with label $i$ and directed outward, then we allow ourselves to write $\epsilon_{i}$ instead of $\epsilon_{f}$.

We next direct the edges of $Q^{*}$ and label each of these directed edges with an element of $\{1, \ldots, n\}$ as follows. Let $e$ be an edge of $Q^{*}$. Let $x$ and $y$ be the vertices of $e$. Theorem 3.1 implies that $x$ and $y$ determine faces $f_{x}$ and $f_{y}$ of the link of the vertex of $M$ and $e$ determines an edge common to $f_{x}$ and $f_{y}$. As in Figure 3 viewed from $f_{x}$, this common edge corresponds to a face $f$ of $Q$ and viewed from $f_{y}$, this common edge corresponds to $f^{-1}$. There exists a unique $i \in\{1, \ldots, n\}$ such that $f \in\left\{F_{i}, F_{i}^{-1}\right\}$. We label $e$ with $i$. If $f=F_{i}$, then we direct $e$ from $x$ to $y$, and if $f=F_{i}^{-1}$, then we direct $e$ from $y$ to $x$.

Example 3.2 (tetrahedron). We give an example to illustrate the construction. Let the model faceted 3-ball $P$ be a tetrahedron with vertices $A, B, C$, and $D$ as in Figure 7 The number 1 which appears in one corner of the face $A B C$ indicates that face $A B C$ has label 1 and is directed outward. The $1^{\prime}$ which appears in one corner of face $A B D$ indicates that face $A B D$ has label 1 and is directed inward. The situation is analogous for faces $A C D$ and $B C D$. The directed circular arcs around $1,1^{\prime}, 2$ and $2^{\prime}$ simply remind us that, as always, faces in figures are oriented clockwise. We proceed as in Section 2 of 4 . We next define face-pairing maps. Face-pairing map $\epsilon_{1}$ maps face $A B C$ to face $A B D$ fixing $A B$, and face-pairing map $\epsilon_{2}$ maps face $A C D$ to face $B C D$ fixing $C D$. We write

$$
\epsilon_{1}:\left(\begin{array}{ccc}
A & B & C \\
A & B & D
\end{array}\right), \quad \epsilon_{2}:\left(\begin{array}{ccc}
A & C & D \\
B & C & D
\end{array}\right)
$$

and $\epsilon=\left\{\epsilon_{1}^{ \pm 1}, \epsilon_{2}^{ \pm 1}\right\}$. The edge cycles for $\epsilon$ have diagrams as follows:

$$
\begin{gathered}
A B \stackrel{\epsilon_{1}}{\rightarrow} A B, \\
B C \stackrel{\epsilon_{1}}{\rightarrow} B D \stackrel{\epsilon_{2}^{-1}}{\rightarrow} A D \stackrel{\epsilon_{1}^{-1}}{\rightarrow} A C \stackrel{\epsilon_{2}}{\rightarrow} B C, \\
C D \stackrel{\epsilon_{2}}{\rightarrow} C D .
\end{gathered}
$$

At present we consider the simplest case and choose each of the edge cycle multipliers to be 1 . Then $A B$ and $C D$ are not subdivided in passing from $P$ to $Q$, but $B C, B D, A D$, and $A C$ are each subdivided into four subedges. Figure 8 shows the faceted 3-ball $Q$. We label the new vertices of $Q$ arbitrarily.

Figure 9 shows the link of the vertex of $M$. For each vertex $v$ of $Q$, there is a face $f(v)$ in this link. The vertices of $f(v)$ are parametrized by the edges of $Q$ incident to $v$; in Figure 9 we label the corners of $f(v)$ by the other vertices incident to those edges. The edges of the link correspond to faces of $Q$, and are labeled 1 or 2 and given a transverse direction accordingly.

Figure 10] shows the faceted 3-ball $Q^{*}$ dual to $Q$. The edge labels and directions of $Q^{*}$ and the vertex labels of $Q^{*}$ are induced by the isomorphism between $\partial Q^{*}$ and the dual of the link of the vertex of $M$. 


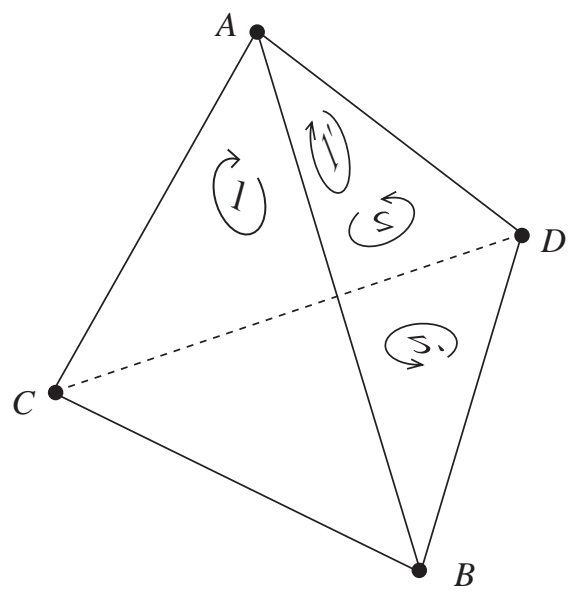

Figure 7. The complex $P$ for the tetrahedron example.

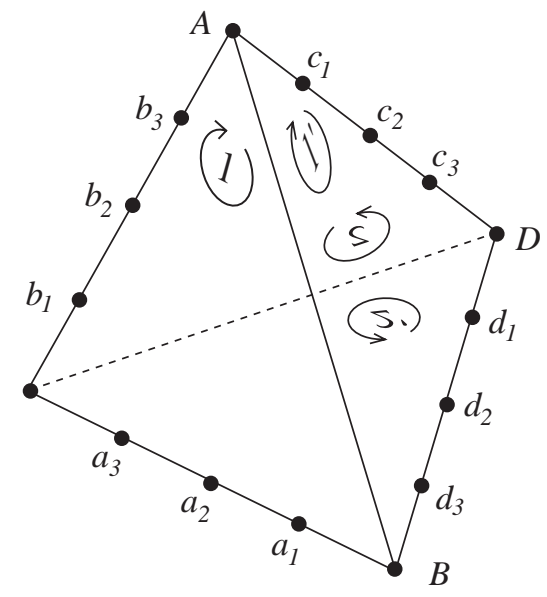

Figure 8. The complex $Q$ for the tetrahedron example.

\section{Duality}

We now wish to construct a twisted face-pairing 3-manifold from $Q^{*}$. It is clear that we may view $Q^{*}$ as a subdivision of $P^{*}$. Furthermore, there exists a facepairing $\epsilon^{*}$ on $P^{*}$ corresponding to $\epsilon$ with multiplier function mul* corresponding to mul so that $Q^{*}$ is gotten from $P^{*}, \epsilon^{*}$ and mul $^{*}$ in the same way that $Q$ is gotten from $P, \epsilon$ and mul. We let $\delta^{*}$ be the twisted face-pairing on $Q^{*}$ determined by $\epsilon^{*}$ and $\mathrm{mul}^{*}$. Note that because the map $\omega: Q \rightarrow Q^{*}$ reverses orientation, $\delta^{*}$ is not the "conjugation" of $\delta$ by $\omega$; the face-pairing $\delta^{*}$ twists in the opposite direction. The following theorem motivates us to consider $\delta^{*}$.

Theorem 4.1. The edge labels and directions of $Q^{*}$ are respected by $\delta^{*}$.

Proof. To prove Theorem 4.1 we further investigate the edge direction and labeling of $Q^{*}$. We return to the setting of paragraph 4 of the proof of Theorem 3.1 With 


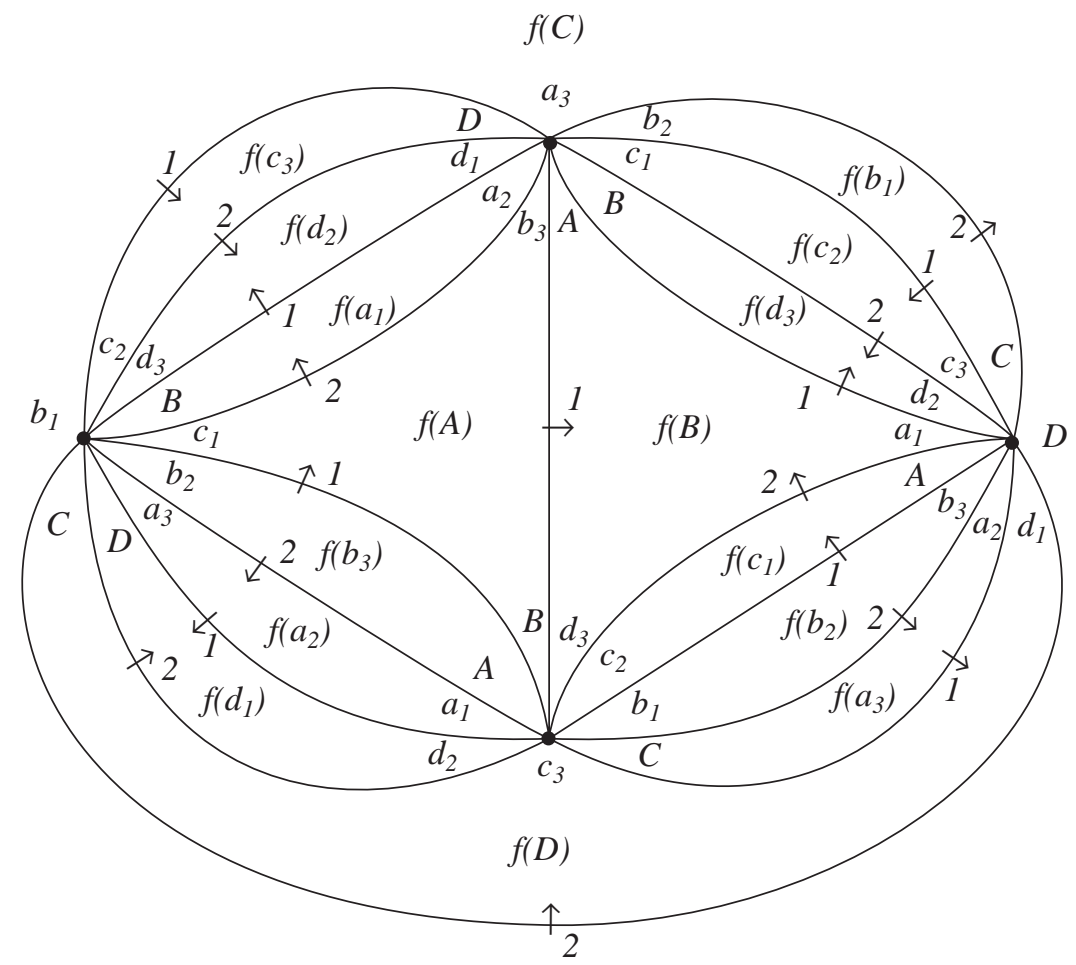

Figure 9. The link of the vertex of $M$.

Figures 5 and 6 in mind, we find it useful to augment Figure 5 with diagonals as in Figure 11. We represent $\omega\left(e_{1}\right)$ as the diagonal from $a_{1}$ to $x_{1}$ to $x_{2}, \ldots$, ending at $x_{\ell_{E} m_{E}}=b_{1}$. For every $i \in\left\{1, \ldots, \ell_{E} m_{E}\right\}$ the edge of $\omega\left(e_{1}\right)$ from $x_{i-1}$ to $x_{i}$ (from $a_{1}$ to $x_{1}$ when $i=1$ ) is labeled with $j$, where $f_{i} \in\left\{F_{j}, F_{j}^{-1}\right\}$ and this edge is directed toward $x_{i}$ if $f_{i}=F_{j}$ and this edge is directed away from $x_{i}$ if $f_{i}=F_{j}^{-1}$. Just as we view $f_{\ell_{E}}^{-1}$ as being left of $e_{1}$ and $f_{1}$ as being right of $e_{1}$, we view $\omega\left(f_{1}\right)$ as being above $\omega\left(e_{1}\right)$ and we view $\omega\left(f_{\ell_{E}}^{-1}\right)$ as being below $\omega\left(e_{1}\right)$. Analogous discussions hold for $\omega\left(e_{2}\right), \ldots, \omega\left(e_{\ell_{E}}\right)$.

Let $i \in\left\{1, \ldots, \ell_{E}\right\}$. It is now clear that applying $\delta_{f_{i}}$ to a vertex of $e_{i}$ other than $b_{i}$ moves that vertex diagonally one unit right and up in Figure 11 Likewise, applying $\delta_{f_{i}}^{*}$ to a vertex of $\omega\left(e_{i}\right)$ other than $a_{i}$ moves that vertex down one unit in Figure 11. Furthermore, just as $\delta_{f_{1}}^{*}\left(b_{1}\right)$ is the vertex below $b_{1}$, it is also true that $\delta_{f_{1}}^{*}\left(a_{1}\right)$ is the vertex below $a_{1}$. Theorem 4.1 follows easily.

Theorem 3.1 implies that $Q^{*}$ and $\delta^{*}$ determine a manifold $M^{*}$ with one vertex the dual of whose link is isomorphic to $\partial Q$ as oriented 2-complexes. We direct the faces of $Q^{*}$ and label them with $1, \ldots, n$ so that $\omega: Q \rightarrow Q^{*}$ preserves directions and labels of faces. We direct the edges of $Q$ and label them with $1, \ldots, n$ in accordance with the isomorphism between $\partial Q$ and the dual of the link of the vertex of $M^{*}$.

Theorem 4.2 (How to label and direct the edges of $Q$ and $Q^{*}$ ). Let e be an edge of $P$. Let $f$ be a face of $P$ which contains $e$, and let $E$ be the edge cycle of $\epsilon$ which contains $e$. Let $F_{1}^{ \pm 1}, \ldots, F_{n}^{ \pm 1}$ be the faces of $P$ so that $F_{k}$ is labeled with $k$ and 


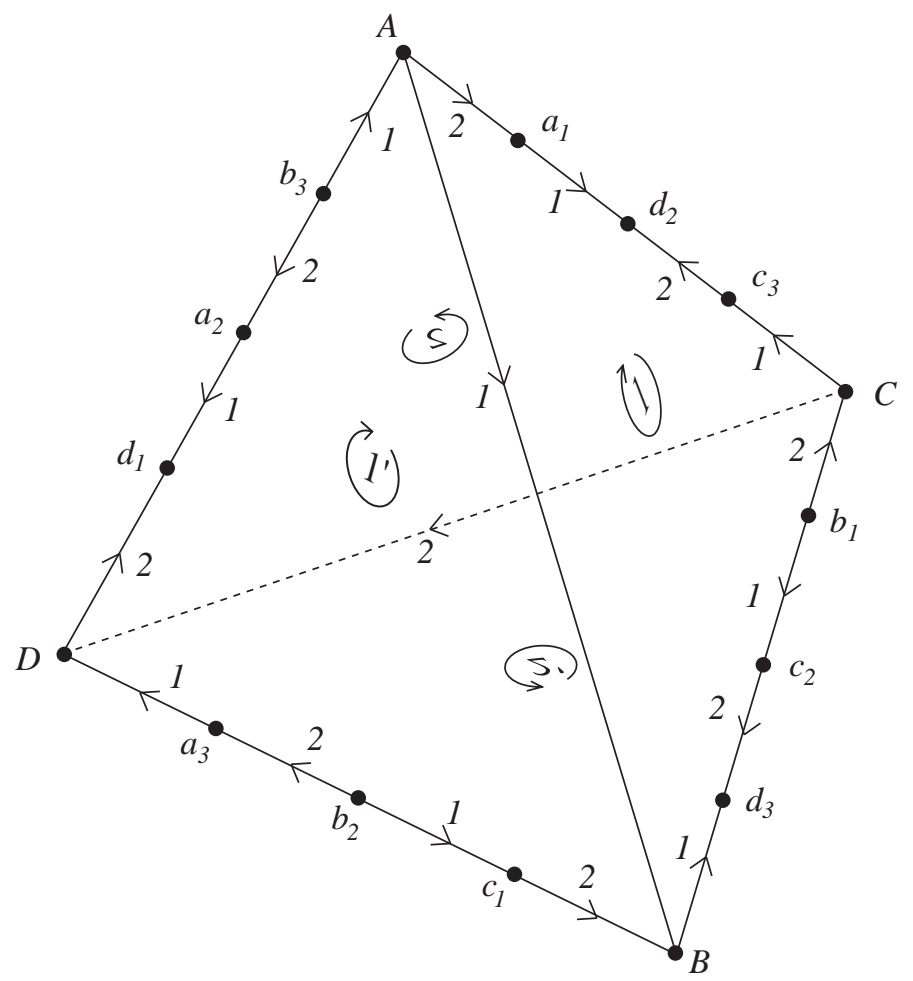

Figure 10. The complex $Q^{*}$ with edge labels and directions.

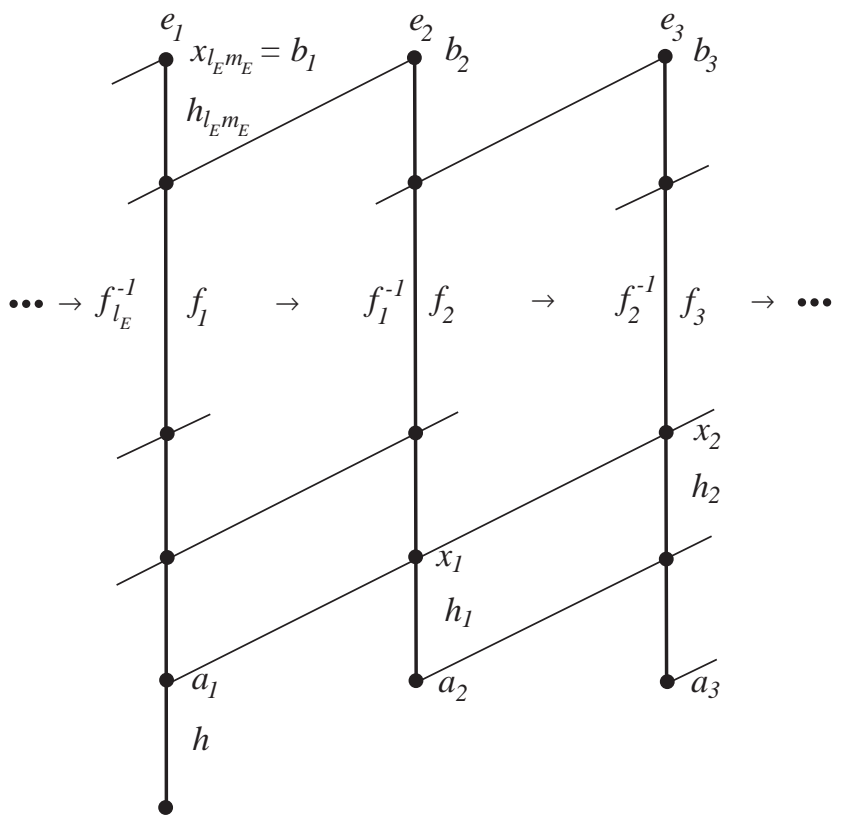

FiguRE 11. Drawing the $\omega\left(e_{i}\right)$ 's as diagonals. 
$f(C)$

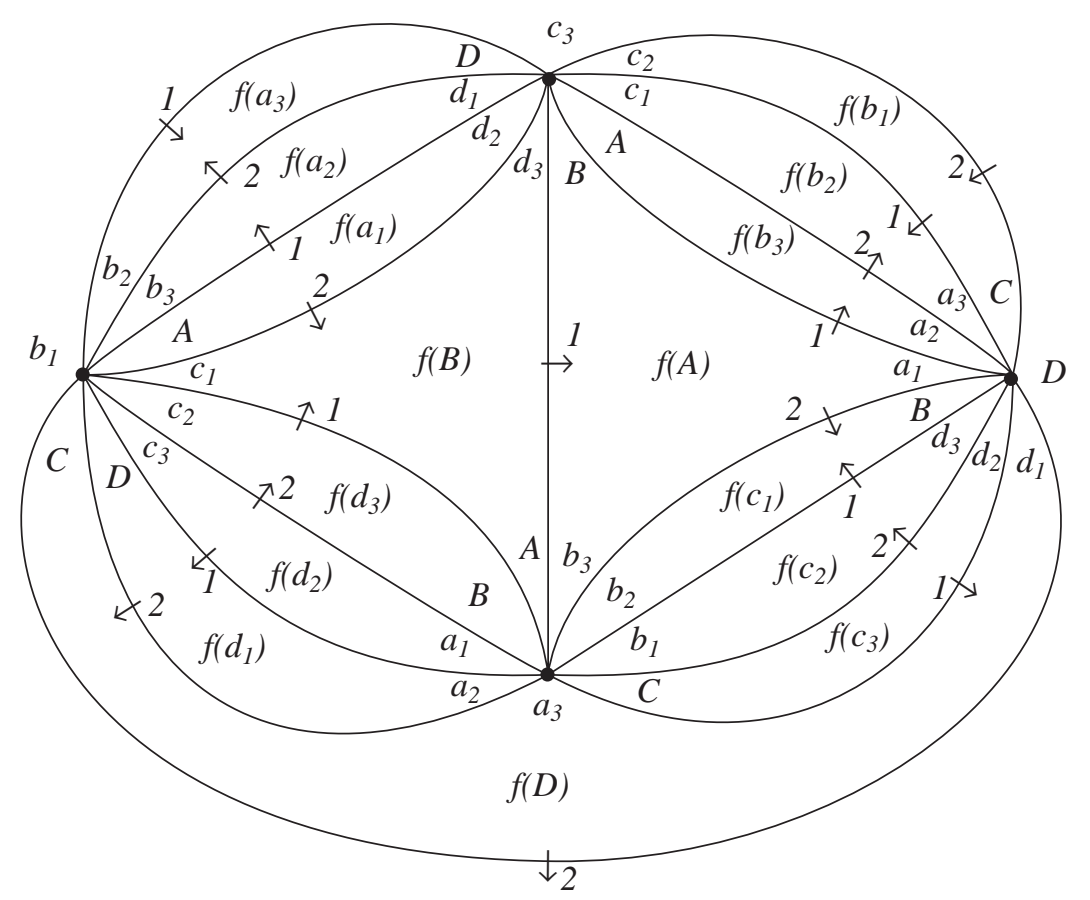

Figure 12. The link of the vertex of $M^{*}$.

directed outward for $k \in\{1, \ldots, n\}$. Let

$$
e_{1} \stackrel{\epsilon_{i_{1}}^{a_{1}}}{\rightarrow} e_{2} \stackrel{\epsilon_{i_{2}}^{a_{2}}}{\rightarrow} \cdots \stackrel{\epsilon_{i_{j-1}}^{a_{j-1}}}{\rightarrow} e_{j} \stackrel{\epsilon_{i_{j}}^{a_{j}}}{\rightarrow} e_{1},
$$

be a diagram of $E$, where $e_{k} \subseteq F_{i_{k}}^{a_{k}}$ and $a_{k} \in\{ \pm 1\}$ for $k \in\{1, \ldots, j\}, e_{1}=e$ and $F_{i_{1}}^{a_{1}}=f$. Let $k \in\{1, \ldots, j\}$. Then, relative to the orientation of $\partial f$, the $(j-k+1)$ th new edge of $e$ in $Q$ has label $i_{k}$ and its direction agrees with the orientation of $\partial f$ exactly when $a_{k}=-1$. Likewise, relative to the orientation of $\partial(\omega(f))$, the $(j-k+1)$ th new edge of $\omega(e)$ in $Q^{*}$ has label $i_{k}$ and its direction agrees with the orientation of $\partial(\omega(f))$ exactly when $a_{k}=-1$. The above description is complete if $\operatorname{mul}(E)=1$. In general, there are mul $(E)$ copies of the above pattern.

Proof. As in the proof of Theorem 4.1 this follows easily from Figure 11.

Example 4.3. We continue with the tetrahedron example of Example 3.2. Just as Figure 9 shows the link of the vertex of $M$, Figure 12 shows the link of the vertex of $M^{*}$. Just as we label and direct the edges of $Q^{*}$ in Figure 10, we label and direct the edges of $Q$ in Figure 13. We can verify that the edge directions and labels of $Q$ are correct by using either Figure 12 or (with much less effort) Theorem 4.2 and (3.3).

Theorem 4.4. Two edges of $Q$ map to the same edge of $M$ if and only if they have the same label. 


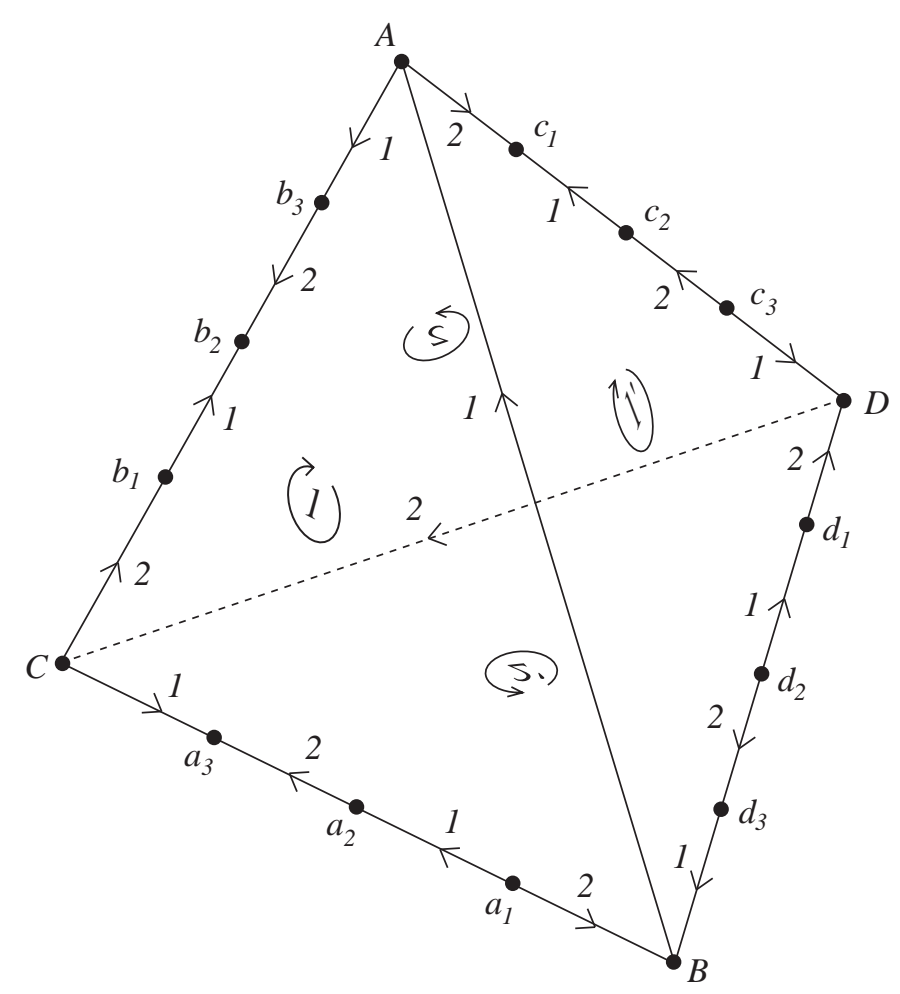

Figure 13. The complex $Q$ with edge labels and directions.

Proof. Theorem 4.1 implies that if two edges of $Q$ map to the same edge of $M$, then they have the same label. On the other hand, the proof of the main theorem of 44 shows that the number of edges of $M$ equals the number of faces of $M$. In other words, the number of edges of $M$ equals the number of edge labels of $Q$. Theorem 4.4 follows.

Corollary 4.5. The directions and labels of the faces and edges of $Q$ induce directions and labels of the faces and edges of $M$ so that $M$ has exactly one face and edge with label $i$ for every $i \in\{1, \ldots, n\}$.

Proof. The assertion for faces is clear, and the assertion for edges follows from Theorem 4.1 and Theorem 4.4

Our next goal is to construct cell complexes $M_{\sigma}$ and $M_{\sigma}^{*}$ which subdivide $M$ and $M^{*}$, respectively, and to show that there exists a cellular homeomorphism from $M_{\sigma}$ to $M_{\sigma}^{*}$.

For this we introduce the notion of a dual cap subdivision. The terminology dual cap subdivision derives from the word dual as in dual complex and the word cap in the meaning of intersection. The dual cap subdivision of a complex is gotten by "intersecting" that complex with its "dual complex". Formally, we next define the dual cap subdivision of a regular CW complex $X$ with dimension at most 3 . We proceed by induction on the dimension of $X$. Suppose that our given complex is an edge $e$, consisting as usual of two 0-cells and one 1-cell. Choose a barycenter 
for $e$, and subdivide $e$ into two edges by means of its barycenter. This determines the dual cap subdivision $e_{\sigma}$ of $e$. In turn this determines the dual cap subdivision $X_{\sigma}$ of $X$ if $X$ has dimension 1. Now suppose that our given complex is a face $f$, consisting of one 2-cell whose boundary is a 1-complex. We have $(\partial f)_{\sigma}$. Choose a barycenter for $f$. We join the barycenter of $f$ with an edge to the barycenter of every edge of $f$. This determines the dual cap subdivision $f_{\sigma}$ of $f$, which subdivides $f$ into quadrilaterals, one for every vertex of $f$. In turn this determines the dual cap subdivision $X_{\sigma}$ of $X$ if $X$ has dimension 2. Finally, suppose that our given complex is a 3-cell $c$ whose boundary is a 2-complex. We have $(\partial c)_{\sigma}$. Choose a barycenter for $c$. We join the barycenter of $c$ with an edge to the barycenter of every face of $c$. To complete our description of $c_{\sigma}$, we describe the faces of the subdivision of $c$ whose interiors lie in the interior of $c$. Let $e$ be an edge of $c$. Let $f$ and $g$ be the faces of $c$ which contain $e$. We have defined edges of $c_{\sigma}$ joining the barycenter of $e$ to the barycenter of $f$, joining the barycenter of $f$ to the barycenter of $c$, joining the barycenter of $c$ to the barycenter of $g$ and joining the barycenter of $g$ to the barycenter of $e$. These four edges of $c_{\sigma}$ determine a face of $c_{\sigma}$ whose interior lies in the interior of $c$. Every face of the subdivision of $c$ whose interior lies in the interior of $c$ has this form. This determines the dual cap subdivision $c_{\sigma}$ of $c$. In turn this determines the dual cap subdivision $X_{\sigma}$ of $X$ if $X$ has dimension 3.

In this paragraph we discuss the structure of the 3-cells which occur in the dual cap subdivision of a 3-cell. For 2-cells the situation is clear: the dual cap subdivision of a 2-cell is a union of quadrilaterals, one for every vertex of the given 2-cell. Figure 14 shows a vertex $v$ of a 2-cell $f$ and parts of the two edges of $f$ which contain $v$, drawn as thick line segments. The dots indicate that part of each edge is not shown. The vertex $u$ is the barycenter of $f$, and the other two vertices are barycenters of the edges of $f$ which contain $v$. We see one of the quadrilaterals in the dual cap subdivision of $f$. Figure 15] is a 3-dimensional version of Figure 14 In Figure 15 $v$ is a vertex of a 3-cell $c$, and the thick line segments which contain $v$ are parts of the edges of $c$ which contain $v$. Now $u$ is the barycenter of $c$. The 3-cell of the dual cap subdivision of $c$ which contains $v$ might be called an alternating suspension; we take an octagon, cone every other vertex of the octagon to $v$ and cone the remaining vertices of the octagon to $u$. In general, suppose that $v$ has valence $k$. Then we cone every other vertex of a $2 k$-gon to $v$, and we cone the remaining vertices of the $2 k$-gon to $u$. Both $u$ and $v$ have valence $k$ in the resulting 3 -cell, and every other vertex has valence 3 .

Having defined the notion of dual cap subdivision, we construct a dual cap subdivision $Q_{\sigma}$ of $Q$ which is $\delta$-invariant. The face-pairing $\delta$ on $Q$ then induces what might be called a face-pairing $\delta_{\sigma}$ on $Q_{\sigma}$. (It is not quite a face-pairing as defined only because $Q_{\sigma}$ has more than one 3-cell.) We define the dual cap subdivision of $M$ to be the cell complex $M_{\sigma}$ of orbits of points of $Q_{\sigma}$ under $\delta_{\sigma}$. We likewise have analogous statements involving $Q_{\sigma}^{*}, \delta_{\sigma}^{*}$ and $M_{\sigma}^{*}$.

Let $C_{1}, \ldots, C_{k}$ be the closed 3-cells of $Q_{\sigma}$. For every $i \in\{1, \ldots, k\}$ let $A_{i}$ be a cell complex isomorphic to $C_{i}$ so that $A_{1}, \ldots, A_{k}$ are mutually disjoint. Let $A$ be the disjoint union of $A_{1}, \ldots, A_{k}$. The face-pairing $\delta_{\sigma}$ on $Q_{\sigma}$ induces in a straightforward way what might be called a face-pairing $\alpha$ on $A$. (This is more general than a face-pairing as defined because $A$ has more than one 3 -cell, these 3 -cells are mutually disjoint and only those faces corresponding to faces in the boundary of $Q_{\sigma}$ are paired with other faces.) The proof of Theorem 3.1 can be viewed as showing that the cell complex which consists of orbits of points of $A$ 


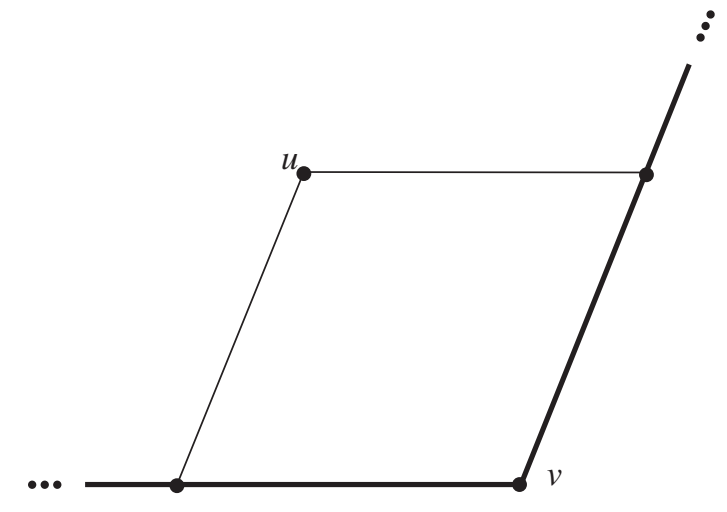

Figure 14. One 2-cell in the dual cap subdivision of a 2-cell.

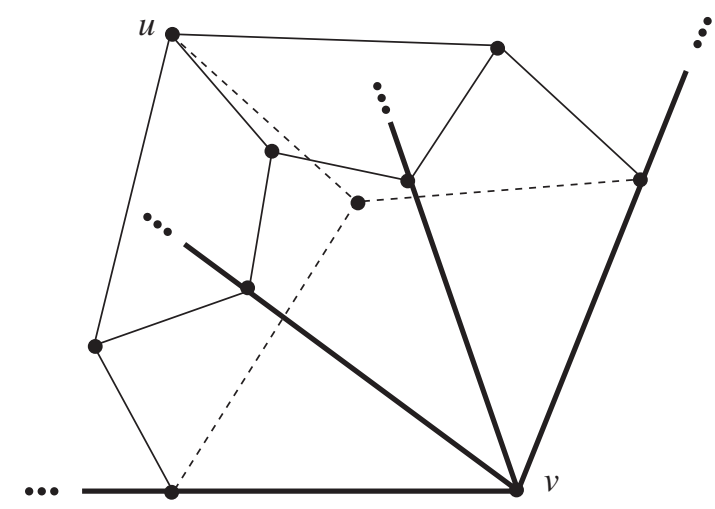

Figure 15. One 3-cell in the dual cap subdivision of a 3-cell.

under $\alpha$ is isomorphic to $Q_{\sigma}^{*}$. Thus we may view $A_{1}, \ldots, A_{k}$ as being cell complexes isomorphic to the 3-cells of $Q_{\sigma}^{*}$. Now the face-pairing $\delta_{\sigma}^{*}$ on $Q_{\sigma}^{*}$ induces a facepairing $\alpha^{*}$ on $A$. By symmetry, the complex which consists of orbits of points of $A$ under $\alpha^{*}$ is isomorphic to $Q_{\sigma}$. It follows that if we begin with $A$, perform the identifications determined by $\alpha$ and then perform the identifications determined by $\delta_{\sigma}^{*}$, we obtain a cell complex isomorphic to $M_{\sigma}^{*}$. So $M_{\sigma}^{*}$ is isomorphic to the cell complex gotten from $A$ by performing the identifications determined by both $\alpha$ and $\alpha^{*}$. Likewise $M_{\sigma}$ is isomorphic to the cell complex gotten from $A$ by performing the identifications determined by both $\alpha$ and $\alpha^{*}$. This proves that there exists a cellular homeomorphism from $M_{\sigma}$ to $M_{\sigma}^{*}$.

Because there exists a cellular homeomorphism from $M_{\sigma}$ to $M_{\sigma}^{*}$, we may view $M$ and $M^{*}$ as giving cellular decompositions of the same space: we take $|M|=\left|M^{*}\right|$. When we do this we see that there exists a duality between the cells of $M$ and the cells of $M^{*}$. The vertex of one corresponds to the 3 -cell of the other. Every edge of one corresponds to a face of the other. In fact, Corollary 4.5 shows that the face and edge labels and directions of $Q$ and $Q^{*}$ determine face and edge labels and directions for $M$ and $M^{*}$. The edge of $M$ labeled with $i \in\{1, \ldots, n\}$ passes through the face of $M^{*}$ labeled with $i$, and the edge of $M^{*}$ labeled with $i \in\{1, \ldots, n\}$ passes 
through the face of $M$ labeled with $i$. Furthermore, directions of edges agree with directions of faces. We summarize this paragraph in the following theorem.

Theorem 4.6. The manifolds $|M|$ and $\left|M^{*}\right|$ are homeomorphic. We may identify them so that the complexes $M$ and $M^{*}$ are dual to each other in a way which respects directions and labels of edges and faces.

Corollary 4.7. Let $P$ be an unoriented faceted 3-ball embedded in $\mathbf{R}^{3}$. Suppose given an orientation-reversing face-pairing on $P$ with a multiplier function. From this we can construct two twisted face-pairings; one twisted face-pairing is gotten by always twisting clockwise and one twisted face-pairing is gotten by always twisting counterclockwise. Then the manifolds gotten from these two twisted face-pairings are homeomorphic.

Proof. This is essentially equivalent to saying that $|M|$ and $\left|M^{*}\right|$ are homeomorphic.

The duality between $M$ and $M^{*}$ motivates us to say that objects such as $\epsilon^{*}, \delta^{*}$, $M^{*}, \ldots$ are dual to the objects $\epsilon, \delta, M, \ldots$.

Let $\widetilde{M}$ be the universal covering cell complex of $M$, and let $\widetilde{M}^{*}$ be the universal covering cell complex of $M^{*}$. We assume that $|M|=\left|M^{*}\right|$, and hence that $|\widetilde{M}|=$ $\left|\widetilde{M}^{*}\right|$. The edge and face directions and labels of $M$ and $M^{*}$ lift to $\widetilde{M}$ and $\widetilde{M}^{*}$. Just as we have a duality between $M$ and $M^{*}$, we have a duality between $\widetilde{M}$ and $\widetilde{M}^{*}$.

Let $G$ be the fundamental group of $M$. We fix an action of $G$ on $\widetilde{M}$. It is clear that $G$ acts cellularly on both $\widetilde{M}$ and $\widetilde{M}^{*}$ and that $G$ preserves edge and face labels and directions. We fix a vertex $\mathcal{O}$ of $\widetilde{M}$, and we call $\mathcal{O}$ the base vertex of $\widetilde{M}$. Likewise we fix a vertex $\mathcal{O}^{*}$ of $\widetilde{M}^{*}$, and we call $\mathcal{O}^{*}$ the base vertex of $\widetilde{M}^{*}$. Let $C$ be the closed 3-cell of $\widetilde{M}$ dual to $\mathcal{O}^{*}$. There exists a canonical cellular map from $Q$ to $C$. Let $F_{1}^{ \pm 1}, \ldots, F_{n}^{ \pm 1}$ denote the faces of $Q$ as before, and let $\widetilde{F}_{1}^{ \pm 1}, \ldots, \widetilde{F}_{n}^{ \pm 1}$ denote their images in $C$ under the canonical map from $Q$ to $C$. Define $n$ elements of $G$ as follows. Let $i \in\{1, \ldots, n\}$. Let $g_{i}$ be the unique element of $G$ such that $\widetilde{F}_{i} \subseteq C \cap g_{i} C$; it follows that $g_{i}$ is the unique element of $G$ such that $\widetilde{F}_{i}^{-1} \subseteq C \cap g_{i}^{-1} C$. We similarly define elements $g_{1}^{*}, \ldots, g_{n}^{*}$ of $G$ using the 3-cell of $\widetilde{M}^{*}$ dual to $\mathcal{O}$.

Theorem 4.8. Let $F_{1}^{ \pm 1}, \ldots, F_{n}^{ \pm 1}$ be the faces of $Q$ as usual. Then the elements $g_{1}, \ldots, g_{n}$ generate $G$, and a defining set of relators for them is determined by the closed edge paths of $Q^{*}$ arising from the boundaries of $\omega\left(F_{1}\right), \ldots, \omega\left(F_{n}\right)$.

Proof. This is standard; for example, see [9, $\S 46$ and $\S 62]$. Let $p: Q^{*} \rightarrow M^{*}$ be the quotient map. The group $G$ is isomorphic to the fundamental group of the spine $p\left(\partial Q^{*}\right)$, and the presentation given by Theorem 4.8 is the standard presentation of the fundamental group of the surface complex $p\left(\partial Q^{*}\right)$.

We refer to $\left\{g_{1}, \ldots, g_{n}\right\}$ as the geometric generating set of $G$.

Example 4.9. We continue with the tetrahedron example of Example 3.2. Let $x_{1}, \ldots, x_{n}$ form a basis of a free group. We consider the diagram of $Q^{*}$ in Figure 10 Beginning with edge $A B$ and proceeding in the counterclockwise direction, the labels and directions of the edges of the face $A B C$ give the relator

$$
x_{1} x_{1} x_{2}^{-1} x_{1}^{-1} x_{2} x_{1} x_{2} x_{1}^{-1} x_{2}^{-1} .
$$


Beginning with edge $C D$ and proceeding in the counterclockwise direction, the labels and directions of the edges of face $A C D$ give the relator

$$
x_{2} x_{2} x_{1}^{-1} x_{2}^{-1} x_{1} x_{2} x_{1} x_{2}^{-1} x_{1}^{-1} .
$$

We conclude that

$$
G \cong\left\langle x_{1}, x_{2}: x_{1} x_{1} x_{2}^{-1} x_{1}^{-1} x_{2} x_{1} x_{2} x_{1}^{-1} x_{2}^{-1}, x_{2} x_{2} x_{1}^{-1} x_{2}^{-1} x_{1} x_{2} x_{1} x_{2}^{-1} x_{1}^{-1}\right\rangle .
$$

Hence $H_{1}(M) \cong G /[G, G]$ is the trivial group, and so $M$ is a homology sphere. We obtain a dual presentation for $G$ by using $Q$ instead of $Q^{*}$ :

$$
G \cong\left\langle x_{1}, x_{2}: x_{1} x_{1} x_{2} x_{1}^{-1} x_{2}^{-1} x_{1} x_{2}^{-1} x_{1}^{-1} x_{2}, x_{2} x_{2} x_{1} x_{2}^{-1} x_{1}^{-1} x_{2} x_{1}^{-1} x_{2}^{-1} x_{1}\right\rangle .
$$

Returning to the general case, we let $\Gamma_{g}$ denote the Cayley graph of $G$ with respect to $\left\{g_{1}, \ldots, g_{n}\right\}$ (the subscript $g$ stands for geometric).

Theorem 4.10. The 1-skeleton of $\widetilde{M}^{*}$ with its labels, directions and base vertex $\mathcal{O}^{*}$ is canonically isomorphic to $\Gamma_{g}$.

Proof. After we map the vertex of $\Gamma_{g}$ represented by the identity element of $G$ to $\mathcal{O}$, Theorem 4.8 easily implies that there exists a unique $G$-equivariant cellular isomorphism from $\Gamma_{g}$ to the 1-skeleton of $\widetilde{M}^{*}$. This proves Theorem 4.10

\section{The Model face-PAIRING PSEUdomanifold $N$ And the group $H$}

Suppose given a faceted 3-ball $P$ with an orientation-reversing face-pairing $\epsilon$. Define $N=N(\epsilon)$ to be the cell complex consisting of the orbits of points of $P$ under $\epsilon$. We call $N$ the model face-pairing pseudomanifold.

Our next goal is to define a group $H$, which is closely related to the fundamental group of $N$. Let $F_{1}^{ \pm 1}, \ldots, F_{n}^{ \pm 1}$ be the faces of $P$, and let $\epsilon_{i}: F_{i} \rightarrow F_{i}^{-1}$ for $i \in\{1, \ldots, n\}$ be face-pairing maps as usual. Let $x_{1}, \ldots, x_{n}$ form a basis of a free group $F$. Let $E$ be an edge cycle of $\epsilon$. Suppose that $E$ has the diagram

$$
e_{1} \stackrel{\epsilon_{i_{1}}^{a_{1}}}{\rightarrow} e_{2} \stackrel{\epsilon_{i_{2}}^{a_{2}}}{\rightarrow} \cdots \stackrel{\epsilon_{i_{j-1}}^{a_{j-1}}}{\rightarrow} e_{j} \stackrel{\epsilon_{i_{j}}^{a_{j}}}{\rightarrow} e_{1}
$$

where $e_{1}, \ldots, e_{j}$ are the edges of $E$ and $a_{1}, \ldots, a_{j} \in\{ \pm 1\}$. To $E$ we associate the element $R_{E}=x_{i_{1}}^{a_{1}} \cdots x_{i_{j}}^{a_{j}} \in F$. The element $R_{E}$ is defined only up to inversion and cyclic permutation, namely, it is defined only up to choosing a diagram for $E$. We define the group $H$ to be given by the presentation

$$
H=\left\langle x_{1}, \ldots, x_{n}:\left\{R_{E}: E \text { is an edge cycle of } \epsilon\right\}\right\rangle .
$$

Theorem 5.1. The map from $\left\{g_{1}, \ldots, g_{n}\right\}$ to $\left\{x_{1}, \ldots, x_{n}\right\}$ given by $g_{i} \mapsto x_{i}$ for $i \in\{1, \ldots, n\}$ determines a surjective group homomorphism from $G$ to $H$.

Proof. Theorems 4.2 and 4.8 show that $G$ is given by a presentation whose relators are products of cyclic permutations of the relators of $H$ and their inverses. Theorem 5.1 follows.

Theorem 5.2. Let $N_{0}$ be the open manifold gotten from $N$ by deleting its vertices. Then $H \cong \pi_{1}\left(N_{0}\right)$. The inclusion $N_{0} \rightarrow N$ gives a surjective group homomorphism $\pi_{1}\left(N_{0}\right) \rightarrow \pi_{1}(N)$ which is an isomorphism if and only if $N$ is a manifold. 
Proof. Let $p: P \rightarrow N$ be the quotient map. By construction $N$ has a natural cell structure induced from the cell structure of $P$. Construct a dual cap subdivision $P_{\sigma}$ of $P$ which is $\epsilon$-invariant. Let $v$ be the barycenter of the 3-cell of $P$, and denote the barycenter of a face $f$ of $P$ by $b(f)$. Let $K$ be the surface complex in $N$ defined as follows. There is exactly one vertex $p(v)$. For each face $f$ of $P$, let $e_{f}$ be the edge in $P_{\sigma}$ joining $v$ and $b(f)$. For each pair $\left\{f, f^{-1}\right\}$ of paired faces in $P$, there is an edge $p\left(e_{f}\right) \cup p\left(e_{f-1}\right)$ in $K$. For each edge $e$ of $P$, there is a face in $K$ which consists of the union of the images under $p$ of the faces in $P_{\sigma}$ which contain $v$ and the barycenter of an edge in the edge cycle of $e$. That is, the vertex of $K$ is dual to the 3 -cell of $N$, the edges of $K$ are dual to the faces of $N$, and the faces of $K$ are dual to the edges of $N$. Then $H \cong \pi_{1}(K)$ (see [9, §46]). Since $K$ is a strong deformation retract of $N_{0}$, we have $\pi_{1}(K) \cong \pi_{1}\left(N_{0}\right)$. If $N$ is a manifold, then $\pi_{1}\left(N_{0}\right) \cong \pi_{1}(N)$. If $N$ is not a manifold, then some vertex of $N$ has a link $F$ which is not simply connected. Let $\alpha$ and $\beta$ be simple closed curves in $F$ which intersect transversely in a single point. It follows easily from duality that the homology classes of $\alpha$ and $\beta$ cannot both be 0 in $H_{1}\left(N_{0}\right)$. Hence the image of $\pi_{1}(F)$ in $\pi_{1}\left(N_{0}\right)$ is nontrivial, and so by van Kampen's theorem $\pi_{1}(N)$ is a proper quotient of $\pi_{1}\left(N_{0}\right)$.

Corollary 5.3. There exists a surjective group homomorphism from $\pi_{1}(M)$ to $\pi_{1}(N)$.

Proof. This follows from Theorem 5.1 and Theorem 5.2.

\section{Homology of $M$ AND $N$}

Since $M$ is a 3 -dimensional cell complex, to $M$ there is associated a chain complex $C(M)$ with boundary operator $\partial$ of the form

$$
0 \longrightarrow C_{3}(M) \stackrel{\partial_{3}}{\longrightarrow} C_{2}(M) \stackrel{\partial_{2}}{\longrightarrow} C_{1}(M) \stackrel{\partial_{1}}{\longrightarrow} C_{0}(M) \longrightarrow 0 .
$$

Likewise we have for $N$ a chain complex $C(N)$ with boundary operator $\partial$ of the form

$$
0 \longrightarrow C_{3}(N) \stackrel{\partial_{3}}{\longrightarrow} C_{2}(N) \stackrel{\partial_{2}}{\longrightarrow} C_{1}(N) \stackrel{\partial_{1}}{\longrightarrow} C_{0}(N) \longrightarrow 0 .
$$

There exists a chain map $\sigma: C(N) \rightarrow C(M)$ with $\sigma_{i}: C_{i}(N) \rightarrow C_{i}(M)$ for $i \in\{0,1,2,3\}$ defined as follows. The map $\sigma_{3}$ maps the 3-cell of $N$ to the 3-cell of $M$. There exists a canonical bijection between the 2-cells of $N$ and the 2-cells of $M$, and $\sigma_{2}$ maps every 2-cell of $N$ to the corresponding 2-cell of $M$. The complex $M$ has only one 0-cell, and $\sigma_{0}$ maps every 0-cell of $N$ to the 0 -cell of $M$. Finally, we discuss the map $\sigma_{1}$. We identify the edges of $N$ with the edge cycles $E_{1}, \ldots, E_{m}$ of $\epsilon$. These edge cycles $E_{1}, \ldots, E_{m}$ form a $\mathbf{Z}$-basis of $C_{1}(N)$. Let $e_{1}, \ldots, e_{n}$ be the edges of $M$, so that $e_{i}$ is labeled with $i$. We view $e_{1}, \ldots, e_{n}$ as forming a $\mathbf{Z}$-basis of $C_{1}(M)$. Let $i \in\{1, \ldots, n\}$, and let $j \in\{1, \ldots, m\}$. Let $x_{i}$ and $R_{E_{j}}$ be as in the definition of $H$ in Section 5. Define $\sigma_{i j}$ to be the sum of the exponents of $x_{i}$ in $R_{E_{j}}$. We set

$$
\sigma_{1}\left(E_{j}\right)=\operatorname{mul}\left(E_{j}\right) \sum_{i=1}^{n} \sigma_{i j} e_{i}
$$

for $j \in\{1, \ldots, m\}$. It remains to orient $e_{i}$ and $E_{j}$. The direction of $e_{i}$ determines the orientation of $e_{i}$. Finally, the choice of edge cycle diagram in defining $R_{E_{j}}$ determines an orientation of $E_{j}$ for which it is possible to prove using Theorem 4.2 that $\sigma_{0}, \sigma_{1}, \sigma_{2}, \sigma_{3}$ give a chain map $\sigma$. 
It is easy to see that we have the following commutative diagram of Abelian groups and group homomorphisms, where the vertical sequences of maps are exact.

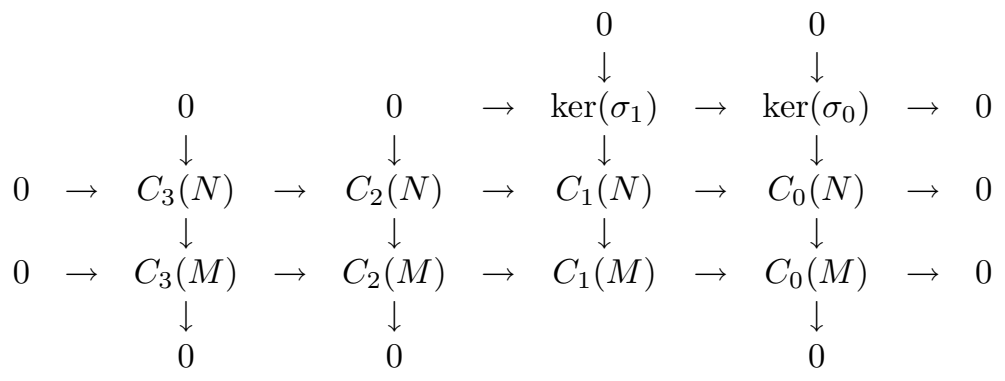

The long exact homology sequence associated to this diagram is

$$
\begin{aligned}
0 \rightarrow H_{3}(N) & \rightarrow H_{3}(M) \rightarrow 0 \rightarrow H_{2}(N) \\
& \rightarrow H_{2}(M) \rightarrow K \rightarrow H_{1}(N) \rightarrow H_{1}(M)
\end{aligned}
$$

where $K$ is the kernel of the homomorphism from $\operatorname{ker}\left(\sigma_{1}\right)$ to $\operatorname{ker}\left(\sigma_{0}\right)$.

Given a finite cell complex $X$, let $b_{i}(X)$ denote its $i$ th Betti number for every nonnegative integer $i$. Statements 4 and 5 are the main statements of the following theorem. The other statements are included for completeness and because they are easy.

Theorem 6.2. We have the following statements.

1. $H_{0}(M) \cong H_{0}(N) \cong H_{3}(M) \cong H_{3}(N) \cong \mathbf{Z}$.

2. $b_{2}(N) \leq b_{2}(M)$.

3. The groups $K, H_{2}(M)$ and $H_{2}(N)$ are free Abelian groups.

4. $b_{1}(M)=\operatorname{rank}(H /[H, H])$.

5. We have $b_{1}(N) \leq b_{1}(M)$, and equality holds if and only if $N$ is a manifold.

6. The image of the map in (6.1) from $H_{1}(N)$ to $H_{1}(M)$ lies in the torsion subgroup of $H_{1}(M)$.

Proof. Statement 1 is clear. Statement 2 is clear because (6.1) shows that $H_{2}(N)$ injects into $H_{2}(M)$. Statement 3 is also clear after noting that both $\partial_{3}: C_{3}(N) \rightarrow$ $C_{2}(N)$ and $\partial_{3}: C_{3}(M) \rightarrow C_{2}(M)$ are zero maps.

Now consider statement 4 . Because $\partial_{1}: C_{1}(M) \rightarrow C_{0}(M)$ is the zero map, $H_{1}(M) \cong C_{1}(M) / \operatorname{im}\left(\partial_{2}\right)$. We next investigate $\partial_{2}: C_{2}(M) \rightarrow C_{1}(M)$. Let $F_{1}, \ldots, F_{n}$ be the usual faces of $Q$, now viewed as forming a $\mathbf{Z}$-basis of $C_{2}(M)$. As usual, the orientation of $Q$ determines orientations of $F_{1}, \ldots, F_{n}$. Let $\Delta$ be the matrix of $\partial_{2}$ with respect to the ordered bases $\left(-F_{1}, \ldots,-F_{n}\right)$ and $\left(e_{1}, \ldots, e_{n}\right)$. Let $\Sigma$ be the matrix of $\sigma_{1}$ with respect to the ordered bases $\left(E_{1}, \ldots, E_{m}\right)$ and $\left(e_{1}, \ldots, e_{n}\right)$. We view the columns of $\Delta$ and $\Sigma$ as vectors in $\mathbf{R}^{n}$. Because $H_{1}(M) \cong$ $C_{1}(M) / \operatorname{im}\left(\partial_{2}\right)$, it follows that $b_{1}(M)$ is the codimension of the $\mathbf{R}$-column space of $\Delta$. The definitions of $H$ and $\sigma_{1}$ easily imply that $\operatorname{rank}(H /[H, H])$ is the codimension of the R-column space of $\Sigma$. Hence to prove statement 4 , it suffices to prove that the column space of $\Delta$ equals the column space of $\Sigma$.

In this paragraph we prove that the column space of $\Delta$ equals the column space of $\Sigma$. Let $j \in\{1, \ldots, n\}$. Using Theorem 4.2 for the first equation, it is not difficult 
to see that

$$
\begin{aligned}
\partial_{2}\left(-F_{j}\right)=\sum_{k=1}^{m} \sigma_{j k} \sigma_{1}\left(E_{k}\right) & =\sum_{k=1}^{m} \sigma_{j k} \operatorname{mul}\left(E_{k}\right) \sum_{i=1}^{n} \sigma_{i k} e_{i} \\
& =\sum_{k=1}^{m} \operatorname{mul}\left(E_{k}\right) \sum_{i=1}^{n} \sigma_{i k} \sigma_{j k} e_{i} .
\end{aligned}
$$

This implies that $\Delta=\sum_{k=1}^{m} \operatorname{mul}\left(E_{k}\right) \Delta_{k}$, where $\Delta_{k}$ is the $n \times n$ matrix whose $i j$ entry is $\sigma_{i k} \sigma_{j k}$ for $k \in\{1, \ldots, m\}$. We prove for $k \in\{1, \ldots, m\}$ that the symmetric matrix $\Delta_{k}$ is positive semidefinite as follows. Let $x=\left(x_{1}, \ldots, x_{n}\right)^{t}$ be a column vector in $\mathbf{R}^{n}$. Then the $i$ th entry of the vector $\Delta_{k} x$ is $\sum_{j=1}^{n} \sigma_{i k} \sigma_{j k} x_{j}$, and so

$$
\begin{aligned}
x^{t} \Delta_{k} x=\sum_{i=1}^{n} x_{i} \sum_{j=1}^{n} \sigma_{i k} \sigma_{j k} x_{j} & =\sum_{i=1}^{n} \sigma_{i k} x_{i} \sum_{j=1}^{n} \sigma_{j k} x_{j} \\
& =\left(\sum_{i=1}^{n} \sigma_{i k} x_{i}\right)^{2} \geq 0 .
\end{aligned}
$$

This proves that $\Delta_{k}$ is positive semidefinite for $k \in\{1, \ldots, m\}$. Hence $\Delta$ is a positive semidefinite symmetric matrix because it is a positive linear combination of positive semidefinite symmetric matrices. It follows that the null space of $\Delta$ is the set of all column vectors $x$ in $\mathbf{R}^{n}$ such that $0=x^{t} \Delta x=\sum_{k=1}^{m} \operatorname{mul}\left(E_{k}\right) x^{t} \Delta_{k} x$. Because $\Delta_{k}$ is positive semidefinite for $k \in\{1, \ldots, m\}$, we have that $\sum_{k=1}^{m} \operatorname{mul}\left(E_{k}\right) x^{t} \Delta_{k} x=$ 0 if and only if $x^{t} \Delta_{k} x=0$ for every $k \in\{1, \ldots, m\}$. In other words, the null space of $\Delta$ is the intersection of the null spaces of $\Delta_{1}, \ldots, \Delta_{m}$. But (6.3) shows that the null space of $\Delta_{k}$ is the orthogonal complement of the line spanned by the column $\left(\sigma_{1 k}, \ldots, \sigma_{n k}\right)^{t}$ for $k \in\{1, \ldots, m\}$. Because $\Delta$ is symmetric, it follows that the orthogonal complement of the column space of $\Delta$ equals the orthogonal complement of the column space of $\Sigma$, and so the column space of $\Delta$ equals the column space of $\Sigma$. This proves statement 4 .

The inequality of statement 5 follows from Corollary 5.3. If $N$ is a manifold, then Theorem 5.2 and statement 4 imply that $b_{1}(N)=b_{1}(M)$. Finally, a classical theorem on Euler characteristics, which is proved in Section 3 of [4], implies that $\chi(N) \geq 0$. In other words, $b_{2}(N) \geq b_{1}(N)$. On the other hand, if $b_{1}(N)=b_{1}(M)$, then $b_{1}(N)=b_{1}(M)=b_{2}(M) \geq b_{2}(N)$, the second equality coming from Poincare duality and the inequality coming from statement 2. It follows that if $b_{1}(N)=$ $b_{1}(M)$, then $b_{1}(N)=b_{2}(N)$, that is, $\chi(N)=0$. As in Section 3 of [4], we have $\chi(N)=0$ if and only if $N$ is a manifold. Thus $b_{1}(N)=b_{1}(M)$ if and only if $N$ is a manifold.

Statement 6 follows from the fact that the column space of $\Delta$ equals the column space of $\Sigma$ : given $x \in C_{1}(N)$ there exists a positive integer $a$ such that $a \sigma_{1}(x) \in$ $\operatorname{im}\left(\partial_{2}\right)$, that is, the homology class of $x$ in $H_{1}(N)$ maps to a homology class in $H_{1}(M)$ whose order divides $a$.

This proves Theorem 6.2.

Corollary 6.4. Let $P$ be a model faceted 3-ball, and let $P^{\prime}$ be a model faceted 3ball gotten from $P$ by subdividing $\partial P$. Let $M$ be the twisted face-pairing manifold gotten from $P$ and some multiplier function, and let $M^{\prime}$ be the twisted face-pairing manifold gotten from $P^{\prime}$ and some multiplier function. Then $b_{1}(M)=b_{1}\left(M^{\prime}\right)$. In particular, $b_{1}(M)$ is independent of the choice of edge cycle multipliers. 
Proof. From statement 4 of Theorem 6.2, it follows that $b_{1}(M)$ is the rank of $H /[H, H]$. Theorem $\left[5.2\right.$ states that $H$ is isomorphic to $\pi_{1}\left(N_{0}\right)$. It is clear that subdividing $\partial P$ does not change $\pi_{1}\left(N_{0}\right)$. Corollary 6.4 follows.

\section{EXAMPLES}

In this section we give several examples of twisted face-pairing 3-manifolds.

Example 7.1 (lens spaces). Let $P$ be a faceted 3-ball with exactly two faces. For convenience we assume that $|P|$ is the unit ball in $\mathbf{R}^{3}$ and that the faces of $P$ are the northern and southern hemispheres. For any orientation-reversing face-pairing $\epsilon$ and multiplier function mul, $M(\epsilon, \mathrm{mul}$ ) is a lens space (and hence has geometry $\left.S^{3}\right)$.

By varying the numbers of sides of the faces, the face-pairing $\epsilon$, and the multiplier function, one can realize many lens spaces as twisted face-pairing 3-manifolds. In 7] we give a different construction of lens spaces as twisted face-pairing 3-manifolds and show that every lens space is a twisted face-pairing 3-manifold.

Example 7.2 (lunes). Let $P$ be a faceted 3 -ball in which each face is a digon. Then $P$ has exactly two vertices. For convenience we assume that $|P|$ is the unit ball in $\mathbf{R}^{3}$, the vertices of $P$ are $(0,0, \pm 1)$, the edges of $P$ are arcs of great circles, and all of the face-pairing maps are isometries.

Let $F$ be the intersection of $P$ with the $x y$-plane. We call $F$ the equatorial disk of $P$. Then $F$ has a cell structure whose vertices are the intersections of $F$ with the edges of $P$ and whose edges are the intersections of $F$ with the faces of $P$. Furthermore, every face-pairing $\epsilon$ on $P$ restricts to an edge pairing on $F$. Conversely, given an edge pairing on a disk $F$ in which no edge is paired with itself, one can construct a faceted 3-ball $P$ with all faces digons and with an orientationreversing face-pairing $\epsilon$ which restricts on the equator of $P$ to an edge pairing of the equatorial disk of $P$ which is equivalent to the given edge pairing on $F$. Since the face-pairing maps are orientation reversing, this can be done in a unique way up to cellular equivalence.

Hence we can describe these examples by giving a disk $F$ with edge pairing. For convenience we label the vertices of $F$ by $\alpha, \beta, \ldots$ and label the corresponding edges of $P$ by $e_{\alpha}, e_{\beta}, \ldots$.

As a simple example, let $F$ be the disk with vertices $\alpha=(1,0,0), \beta=(0,1,0)$, $\gamma=(-1,0,0)$, and $\delta=(0,-1,0)$, and with edge pairing the antipodal map. Then $P$ has four lunes, and $\epsilon$ is the antipodal map. See Figure 16. We label and direct the faces of $P$ as in Figure 16. The two edge cycles of $\epsilon$ have diagrams

$$
e_{\alpha} \stackrel{\epsilon_{1}}{\rightarrow} e_{\gamma} \stackrel{\epsilon_{2}^{-1}}{\rightarrow} e_{\alpha} \quad \text { and } \quad e_{\beta} \stackrel{\epsilon_{2}}{\rightarrow} e_{\delta} \stackrel{\epsilon_{1}}{\rightarrow} e_{\beta}
$$

Choose each of the multipliers to be 1 . Then each edge of $P$ is subdivided into two edges in $Q$, so each of the four faces of $Q$ is a quadrilateral. See Figure 17

Next we prepare to construct the universal cover $\widetilde{M}$ of $M$. To begin this, take a regular octahedron in $\mathbf{R}^{3}$ with $(0,0, \pm 1)$ as a pair of opposite vertices. For future convenience we assume that each of the octahedron's edges in the $x y$-plane is parallel to either the $x$-axis or the $y$-axis. Now remove the edges in the $x y$-plane. Instead of having eight triangles as faces, we now have four quadrilaterals as faces. This cell complex $Q^{\prime}$ is isomorphic to $Q$. The midpoints of the eight edges of $Q^{\prime}$ are the 


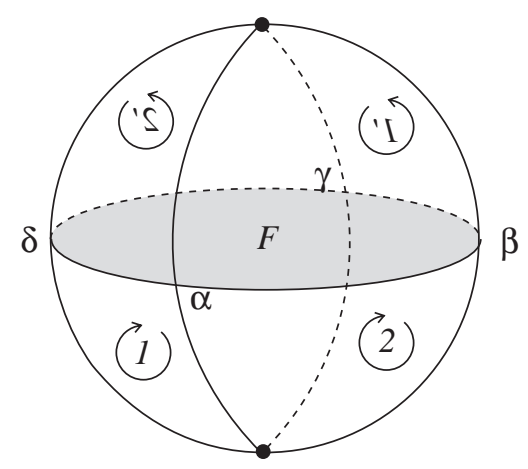

Figure 16 . The model faceted 3 -ball $P$ and its equatorial disk $F$.

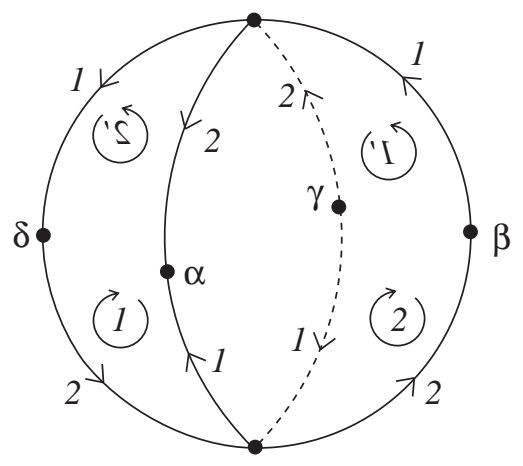

Figure 17. The faceted 3-ball $Q$.

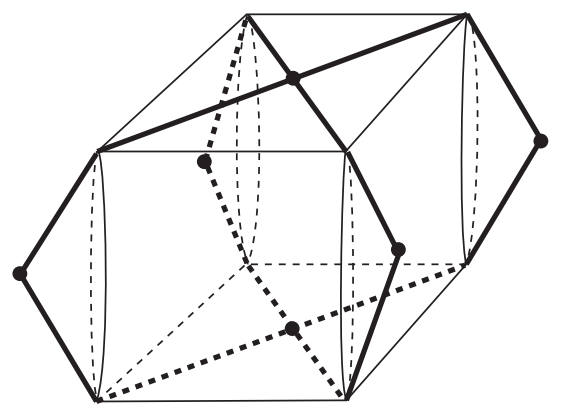

Figure 18. The cube-with-fins $C$.

vertices of an inscribed cube. The horizontal edges of this cube are on the boundary of $Q^{\prime}$. Now deform $Q^{\prime}$, without changing its cell structure, by flattening the parts of $Q^{\prime}$ above and below the cube to the cube. Next deform $Q^{\prime}$ further by flattening the sides of $Q^{\prime}$ toward the sides of this cube. Then $Q^{\prime}$, and hence $Q$, becomes a "cube-with-fins", which we denote by $C$. See Figure 18, where the edges of $C$ are drawn in bold.

Figure 19 shows a pair of nested cubes in $\mathbf{R}^{3}$. By adding arcs of diagonals joining corresponding vertices of the two nested cubes, one gets a decomposition of 


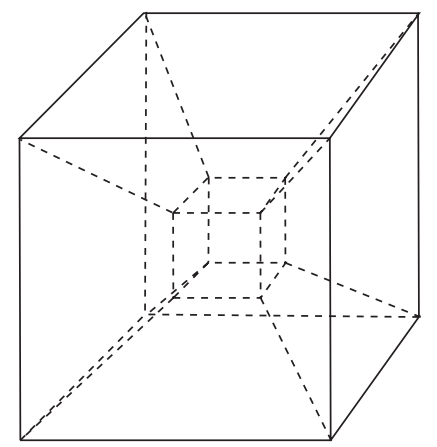

Figure 19. Decomposing $S^{3}$ into eight cubes.

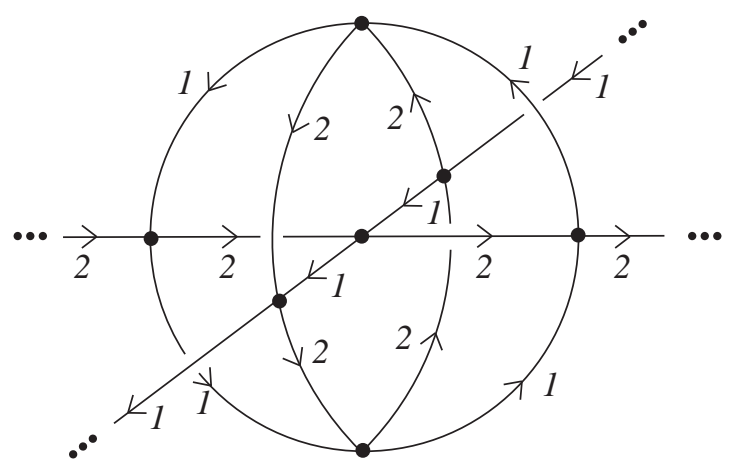

Figure 20. The 1-skeleton of $\widetilde{M}^{*}$.

the larger cube into seven 3-cells, each of which is cellularly isomorphic to a cube. By switching from $\mathbf{R}^{3}$ to $S^{3}$, one gets a cellular decomposition of $S^{3}$ into eight cubes.

In this paragraph we construct $\widetilde{M}$ and show that the 1 -skeleton of $\widetilde{M}^{*}$ is isomorphic to the 1-complex in Figure 20, which has one vertex at infinity. For this we begin with our cube-with-fins $C$, which is cellularly isomorphic to $Q$. Although $C$ is a cube-with-fins and not a cube, we view $C$ as corresponding to the central cube in Figure [19] We label and direct the four faces of $C$ so that the face which meets the positive $x$-axis has label 1 and is directed outward, the face which meets the negative $x$-axis has label 1 and is directed inward, the face which meets the positive $y$-axis has label 2 and is directed outward, and the face which meets the negative $y$-axis has label 2 and is directed inward. The central vertex in Figure 20 is dual to $C$ and the four edges in Figure 20 which contain the central vertex are dual to the four faces of $C$ in a way which respects labels and directions. Now we take a copy of $C$ and attach the face of the copy with label 1 directed inward to the face of $C$ with label 1 directed outward according to our twisted face-pairing. The vertex on the positive $x$-axis in Figure 20 is dual to this copy of $C$. It is easy to check that the four edges in Figure 20 which meet the positive $x$-axis are dual to the four faces of this copy of $C$ in a way which respects labels and directions. We attach three more 
copies of $C$ to $C$ in the same way. We now have five cubes-with-fins corresponding to five of the cells in Figure 19, Next it is not difficult to see using Figure 20 that we can take another copy of $C$, place it above $C$ and attach each of its four faces according to our twisted face-pairing to the top face of one of the four copies of $C$ which we attached to $C$. We likewise take a copy of $C$, place it below $C$ and attach each of its four faces according to our twisted face-pairing to the bottom face of one of the four copies of $C$ which we attached to $C$. Our seven cubes-with-fins are the 3-cells in a cellular decomposition of a closed 3-ball. These cells are arranged like the cells in Figure 19, but our complex of cubes-with-fins is not the complex in Figure 19. Finally, it is not difficult to see that we can attach one more copy of $C$ to our complex of cubes-with-fins according to our twisted face-pairing to obtain a cellular decomposition of $S^{3}$. Thus we have just constructed $\widetilde{M}$. We see that $|\widetilde{M}|$ is homeomorphic to $S^{3}$ and that the 1-skeleton of $\widetilde{M}^{*}$ is isomorphic to the 1-complex in Figure 20.

In this paragraph we identify $G=\pi_{1}(M)$. We get a faceted 3-ball $Q^{*}$ dual to $Q$ by reflecting $Q$ in the $x y$-plane. This and Theorem 4.2 show that we may take $Q^{*}=Q$ as oriented, directed and labeled complexes. Theorem 4.8 implies that with respect to our geometric generating set $\left\{g_{1}, g_{2}\right\}$, we have

$$
G \cong\left\langle x_{1}, x_{2}: x_{1} x_{2} x_{1} x_{2}^{-1}, x_{2} x_{1}^{-1} x_{2} x_{1}\right\rangle,
$$

where $g_{1} \mapsto x_{1}$ and $g_{2} \mapsto x_{2}$. It is easy to see that there exists a surjective group homomorphism from the latter group to the quaternion group $\{ \pm 1, \pm i, \pm j, \pm k\}$ of order 8 with $x_{1} \mapsto i$ and $x_{2} \mapsto j$. The previous paragraph implies that $|G|=8$, and so $G$ is isomorphic to the quaternion group of order 8. As assured by Theorem 4.10 we see that Figure 20 gives a Cayley graph for $G$.

Now consider using the same faceted 3-ball $P$ with four lunes, but using as facepairing $\epsilon$ the orientation-reversing maps which take each face to the opposite face and fix the vertices $(0,0, \pm 1)$. There is a single edge cycle. Given a positive integer $m$, let mul be the multiplier function which assigns $m$ to this edge cycle. Using the surgery description in [6], it is easy to see that $M(\epsilon, \mathrm{mul})$ is obtained by Dehn surgery on the Borromean rings with framings 0,0 , and $1 / m$. When $m=1$ this is the Heisenberg manifold (the prototypical Nil manifold).

In [7] we will show that every twisted face-pairing manifold which is obtained from a model faceted 3-ball whose faces are all digons is a Seifert fibered manifold whose base surface is the orbit space of its equatorial disk with edge pairing. In the above case of the antipodal map on a square, the quotient surface is the projective plane, which is consistent with the fact that our twisted face-pairing manifold has the geometry of $S^{3}$. In the above case of the square with edge pairing which takes each edge to the opposite edge by translation, the quotient surface is the torus, which is consistent with the fact that our twisted face-pairing manifold is the Heisenberg manifold.

Example 7.3. Let $P$ be the unit ball in $\mathbf{R}^{3}$, decomposed into a faceted 3-ball as follows. See Figure 21. There are three vertices, $A=(-1,0,0), B=(0,1,0)$, and $C=(0,-1,0)$. The three $\operatorname{arcs} A B, B C$, and $A C$ on the equator are edges of $P$. There are two edges $n_{A B}$ and $n_{A C}$ in the northern hemisphere; $n_{A B}$ joins $A$ and $B$ and $n_{A C}$ joins $A$ and $C$. The images $s_{A B}$ and $s_{A C}$ of $n_{A B}$ and $n_{A C}$ under the reflection in the $x y$-plane are also edges. These are all of the edges. There are six faces in $P$; there are two digons and a triangle in the northern hemisphere and 


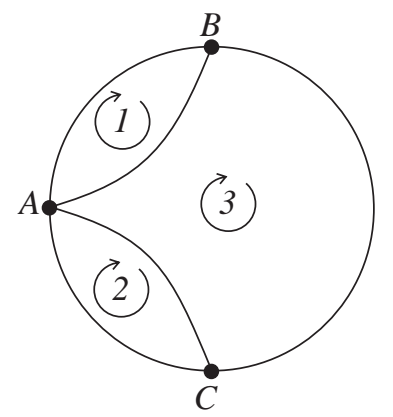

Figure 21. The northern hemisphere of $P$.
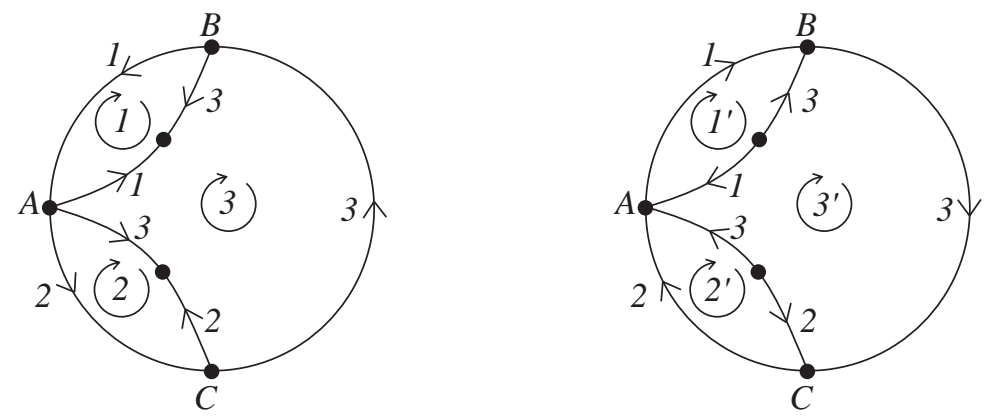

FiguRE 22. The northern hemispheres of $Q$ and $Q^{*}$.

there are two digons and a triangle in the southern hemisphere. Each face is paired with its reflection in the $x y$-plane, and each of the face-pairing maps is given by reflection in the $x y$-plane. We label and direct the faces of $P$ as in Figure 21.

The edge cycles of the model face-pairing are $\{A B\},\{B C\},\{A C\},\left\{n_{A B}, s_{A B}\right\}$, and $\left\{n_{A C}, s_{A C}\right\}$. Figure 22 shows the northern hemispheres of $Q$ and $Q^{*}$ (gotten from $Q$ by reflection in the $x y$-plane) for the multiplier function which assigns 1 to every edge cycle. In general, given a multiplier function mul, let $p=$ $\operatorname{mul}(\{A B\}), q=\operatorname{mul}(\{A C\}), r=\operatorname{mul}(\{B C\}), s=\operatorname{mul}\left(\left\{n_{A B}, s_{A B}\right\}\right)$, and $t=$ $\operatorname{mul}\left(\left\{n_{A C}, s_{A C}\right\}\right)$. Then Theorem 4.8 shows that, with respect to our geometric generating set $\left\{g_{1}, g_{2}, g_{3}\right\}$,

$$
G \cong\left\langle x_{1}, x_{2}, x_{3}:\left(x_{1}\right)^{p}\left(x_{3}^{-1} x_{1}\right)^{s},\left(x_{2}\right)^{q}\left(x_{3}^{-1} x_{2}\right)^{t},\left(x_{3}\right)^{r}\left(x_{2}^{-1} x_{3}\right)^{t}\left(x_{1}^{-1} x_{3}\right)^{s}\right\rangle,
$$

where $g_{1} \mapsto x_{1}, g_{2} \mapsto x_{2}$ and $g_{3} \mapsto x_{3}$.

First suppose $s=t=1$. Then $g_{3}=g_{1}^{p+1}, g_{3}=g_{2}^{q+1}$, and $1=g_{3}^{r} g_{2}^{-1} g_{3} g_{1}^{-1} g_{3}=$ $g_{2}^{-1} g_{3} g_{1}^{-1} g_{3}^{r+1}$. This implies that $g_{2}=g_{1}^{(p+1)(r+1)+p}, g_{1}^{p+1}=g_{1}^{(q+1)((p+1)(r+1)+p)}$, and $G \cong \mathbf{Z} /((p+1)(q+1)(r+1)+p q-1) \mathbf{Z}$. Using the results of [6], it can be shown that $M$ is homeomorphic to the lens space $L((p+1)(q+1)(r+1)+p q-$ $1, q r+2 q+r+1)$.

For general values of $p, q, r, s$, and $t$,

$$
G \cong\left\langle x_{1}, x_{3}:\left(x_{1}\right)^{p}\left(\left(x_{3}\right)^{-1} x_{1}\right)^{s}\right\rangle *\langle a, b\rangle\left\langle x_{2}, x_{3}:\left(x_{2}\right)^{q}\left(\left(x_{3}\right)^{-1} x_{2}\right)^{t}\right\rangle,
$$




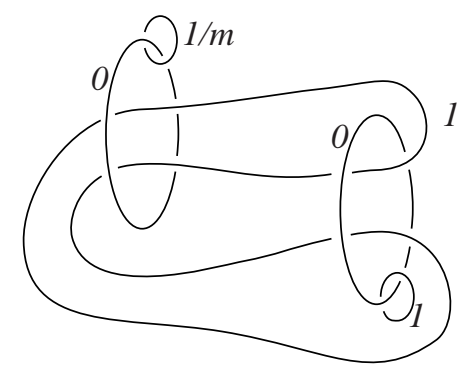

Figure 23. A surgery description for $M(\epsilon$, mul).

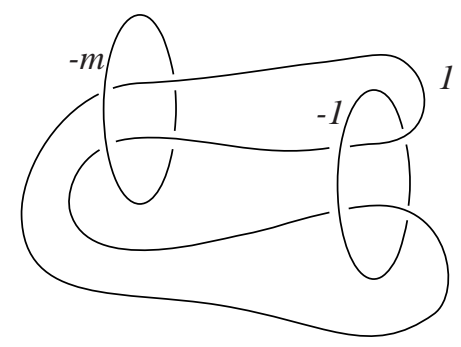

FIgURE 24. A second surgery description for $M(\epsilon$, mul).

where in the left homomorphism $a \mapsto x_{3}$ and $b \mapsto\left(x_{1}\right)^{p}\left(x_{3}\right)^{r}$, and in the right homomorphism $a \mapsto x_{3}$ and $b \mapsto\left(x_{2}\right)^{-q}$. Note that because $g_{1}^{p}=\left(g_{1}^{-1} g_{3}\right)^{s}$, it follows that $g_{1}^{-1} g_{3}$ commutes with $g_{1}^{p}$. Hence $g_{3}$ commutes with $g_{1}^{p}$. It follows that the image of $a$ in $G$ commutes with the image of $b$, and so the above amalgamation is along an Abelian group.

If $p=q=s=t=2$, then $G$ is a Solv group for every choice of $r$. Using the results of [6], it can be shown that if $p=q=s=t=2$, then $M$ is a Solv manifold for every choice of $r$.

Example 7.4 (cube). Let $P$ be a cube in $\mathbf{R}^{3}$ with center at the origin. Pair each face of $P$ with its opposite face, and let each face-pairing map be the antipodal map.

Then $P$ is the simplest example of an ample faceted 3-ball, and with this choice of model face-pairing $\epsilon$ each edge cycle has length two. We choose each multiplier to be 1 . Then $Q$ is obtained from the cube $P$ by subdividing each edge of $P$ into two edges. Let $M=M(\epsilon, \mathrm{mul})$, and let $G=\pi_{1}(M)$. We know from [5] that $G$ is Gromov hyperbolic. According to SnapPea [12], $M$ is hyperbolic. The volume of $M$ is about 5.3335, and the shortest geodesic in $M$ has length about 1.2659. Since the face-pairing $\epsilon$ is preserved by the group of symmetries of the cube, $M$ has that group as a subgroup of its group of symmetries. According to SnapPea, this group of order 48 is the symmetry group of $M$.

Example 7.5 (hexahedron). For this example, let $P$ be a hexahedron in $\mathbf{R}^{3}$ which is the union of two regular tetrahedra along a common face. We assume that $P$ is centered at the origin and that its three vertices of valence 4 are in the $x y$-plane. 
Let $\epsilon$ be the model face-pairing for which each face is paired to its image under reflection in the $x y$-plane and each face-pairing map is reflection in the $x y$-plane.

The three edges in the $x y$-plane are in edge cycles of length 1 , and each of the other three edge cycles has length 2 . We begin by choosing a multiplier function mul so that the multiplier is 2 if the edge cycle has length 1 and is 1 if the edge cycle has length 2. So each edge of $P$ is subdivided into two edges in $Q$, and each of the six faces of $Q$ is a hexagon. By SnapPea, $M(\epsilon, \mathrm{mul})$ is a hyperbolic 3-manifold with volume approximately 1.8319 . Now let $\mathrm{mul}^{\prime}$ be the constant multiplier function with each multiplier 2. Again, by SnapPea, $M\left(\epsilon, \mathrm{mul}^{\prime}\right)$ is a hyperbolic 3-manifold with volume approximately 5.1379 and symmetry group of order 48 .

Example 7.6. We return to the tetrahedron example of Example 3.2. In Section 3 we choose for multiplier function the constant function 1, and in Example 4.9 we show that for this choice of multiplier function $M$ is a homology sphere. More generally, suppose $m$ is a positive integer and define the multiplier function mul by $\operatorname{mul}(\{A B\})=1, \operatorname{mul}(\{C D\})=m$, and $\operatorname{mul}(\{B C, B D, A D, A C\})=1$. Using the surgery description in [6], one sees that $M(\epsilon$, mul $)$ is given by Dehn surgery on the framed link in $S^{3}$ shown in Figure 23. Fenn-Rourke moves on the two components which link the components with framing 0 and give an equivalent surgery description in Figure 24 More Fenn-Rourke moves reduce the framed link of Figure 24 to the figure eight knot with framing $-m$, which is equivalent to the figure eight knot with framing $m$. Hence $M(\epsilon, \mathrm{mul})$ is obtained from the $(m, 1)$ Dehn filling on the figure eight knot complement. At the end of Section 6 of [4 we related $M(\epsilon, \mathrm{mul})$ to the figure eight knot in a rather different way using partial twisted face-pairings. If $m=1$, then $M(\epsilon, \mathrm{mul})$ is the Brieskorn homology sphere $\Sigma(2,3,7)$, which has the geometry of the universal cover of $P S L(2, \mathbf{R})$. According to [10. Theorem 4.7], $M(\epsilon, \mathrm{mul})$ is hyperbolic if $m \geq 5$.

\section{REFERENCES}

[1] J. W. Cannon, The combinatorial Riemann mapping theorem, Acta Math. 173, (1994) 155234. MR 95k:30046

[2] J. W. Cannon, W. J. Floyd, and W. R. Parry, Squaring rectangles: the finite Riemann mapping theorem, in The Mathematical Heritage of Wilhelm Magnus-Groups, Geometry and Special Functions, Contemporary Mathematics 169, (Amer. Math. Soc., Providence 1994) 133-212. MR 95g:20045

[3] J. W. Cannon, W. J. Floyd, and W. R. Parry, Sufficiently rich families of planar rings, Ann. Acad. Sci. Fenn. 24 (1999) 265-304. MR 2000k:20057

[4] J. W. Cannon, W. J. Floyd, and W. R. Parry, Introduction to twisted face-pairings, Math. Res. Lett. 7 (2000), 477-491. CMP 2001:01

[5] J. W. Cannon, W. J. Floyd, and W. R. Parry, Ample twisted face-pairing 3-manifolds, preprint.

[6] J. W. Cannon, W. J. Floyd, and W. R. Parry, Heegaard diagrams and surgery descriptions for twisted face-pairing 3-manifolds, preprint.

[7] J. W. Cannon, W. J. Floyd, and W. R. Parry, A survey of twisted face-pairing 3-manifolds, in preparation.

[8] J. W. Cannon and E. L. Swenson, Recognizing constant curvature discrete groups in dimension 3, Trans. Amer. Math. Soc. 350 (1998) 809-849. MR 98i:57023

[9] H. Seifert and W. Threlfall, Lehrbuch der Topologie, (Chelsea Publishing Company, New York 1947).

[10] W. P. Thurston, The Geometry and Topology of 3-Manifolds, Princeton lecture notes, http://www.msri.org/gt3m, 1979. 
[11] W. P. Thurston, Three-Dimensional Geometry and Topology, Vol. 1, (Princeton University Press, Princeton 1997). Edited by S. Levy. MR 97m:57016

[12] J. Weeks, SnapPea: A computer program for creating and studying hyperbolic 3-manifolds, http://www.northnet.org/weeks.

Department of Mathematics, Brigham Young University, Provo, Utah 84602

E-mail address: cannon@math.byu.edu

Department of Mathematics, Virginia Tech, Blacksburg, Virginia 24061

E-mail address: floyd@math.vt.edu

$U R L:$ http://www.math.vt.edu/people/floyd

Department of Mathematics, Eastern Michigan University, Ypsilanti, Michigan 48197

E-mail address: walter.parry@emich.edu 